QUARTERLY OF APPLIED MATHEMATICS

VOLUME LXVI, NUMBER 2

JUNE 2008, PAGES 249-279

S 0033-569X(08)01103-9

Article electronically published on February 8, 2008

\title{
EXISTENCE OF THE UNIQUE STRONG SOLUTION FOR A CLASS OF NON-NEWTONIAN FLUIDS WITH VACUUM
}

\author{
BY
}

XIAOJING XU (Sch. Math. Sci. \& Lab. Math. Com. Sys., Beijing Normal University, Beijing, 100875, China, and Institute of Mathematics, Jilin University, Changchun, Jilin, 130012, China)

AND

HONGJUN YUAN (Institute of Mathematics, Jilin University, Changchun, Jilin, 130012, China)

Abstract. The aims of this paper are to discuss local existence and uniqueness of solutions for a class of non-Newtonian fluids with vacuum in one-dimensional bounded intervals. The important point in this paper is that we allow the initial vacuum.

1. Introduction and main results. In this paper, we consider a class of compressible non-Newtonian fluids with vacuum in one-dimensional bounded intervals:

$$
\left\{\begin{array}{l}
\rho_{t}+(\rho u)_{x}=0 \quad(x, t) \in(0,1) \times(0, T), \\
(\rho u)_{t}+\left(\rho u^{2}\right)_{x}-\left[\left(u_{x}^{2}+\mu_{0}\right)^{\frac{p-2}{2}} u_{x}\right]_{x}+\pi_{x}=\rho f,
\end{array}\right.
$$

with the initial and boundary conditions

$$
\begin{cases}\left.(\rho, u)\right|_{t=0}=\left(\rho_{0}, u_{0}\right) & x \in[0,1], \\ \left.u\right|_{x=0}=\left.u\right|_{x=1}=0 & t \in[0, T],\end{cases}
$$

where $\rho, u$, and $\pi$ denote the unknown density, velocity, and pressure, respectively. The motion of fluids is driven by an external force $f$, the initial density $\rho_{0} \geqslant 0$, and $p>2$, $\mu_{0}>0$ are both given constants. $\Omega_{T}=I \times(0, T), I=(0,1)$.

Fluid dynamics has attracted the attention of many mathematicians and engineers. The Navier-Stokes equations are generally accepted as the governing equations for the compressible or incompressible motion of viscous fluids, which is usually described by the principle of conservations of mass and momentum, and deduced as

$$
\left\{\begin{array}{l}
\rho_{t}+\operatorname{div}(\rho u)=0 \\
(\rho u)_{t}+\operatorname{div}(\rho u \otimes u)-\operatorname{div}(\Gamma)+\nabla \pi=\rho f .
\end{array}\right.
$$

Received May 16, 2006.

2000 Mathematics Subject Classification. Primary 76A05, 76N10.

Key words and phrases. Existence and uniqueness, non-Newtonian fluid, vacuum.

Supported by program 985 of Jilin University; China Postdoctoral Sciences Foundation; NSF Grants [10571072] and [10601009]; Program 973 [2006cb805900].

E-mail address: xjxu@bnu.edu.cn

E-mail address: hjy@jlu.edu.cn

(C)2008 Brown University 
In 1970, O. A. Ladyzhenskaya (see [1]) proposed a special form for $\Gamma$ :

$$
\Gamma_{i j}=\left(\mu_{0}+\mu_{1}\left|E_{i j}(\nabla u)\right|^{p-2}\right) E_{i j}(\nabla u) .
$$

These models are called

$$
\left\{\begin{array}{lrl}
\text { Newtonian } & \text { for } \mu_{0}>0, \mu_{1}=0, & \\
\text { Ostwald }- \text { de Waele } & \text { for } \mu_{0}=0, \mu_{1}>0, & p>1, \\
\text { Rabinowitsch } & \text { for } \mu_{0}, \mu_{1}>0, & p=4, \\
\text { Eills } & \text { for } \mu_{0}, \mu_{1}>0, & p>2, \\
\text { Bingham } & \text { for } \mu_{0}, \mu_{1}>0, & p=1,
\end{array}\right.
$$

where the values of the parameters $\mu_{1}, p$ in some of the pseudo-plastic Ostwald-de Waele models are given in Whitaker [2]. For example, for paper pulp, $\mu_{1}=0.418, p=1.575$, and for carboxymethyl cellulose in water, $\mu_{1}=0.194, p=1.566$. For more physical meaning, see [3, 4].

In this paper, we study local existence and uniqueness of solutions to the initial boundary value problem (1.1)-(1.2) with nonnegative initial densities. In the case that the data $\rho_{0}, u_{0}, f$ are sufficiently regular and the initial density $\rho_{0}$ has a positive lower bound, there exists a unique measure-valued or weak solution to the problem (1.3), (1.2). For details, we refer the readers to the papers [5, 6, 7, 8, 9, 10,

On the other hand, for the Newtonian fluid, namely $p=2$, there have been few existence results on the solutions for the general case of nonnegative initial densities. The first result was proved by R. Salvi and L. Straskraba. They showed in [1] that if $\Omega$ is a bounded domain in $\mathbb{R}^{3}, \pi=\pi(\cdot) \in C^{2}[0, \infty), \rho_{0} \in H^{2}(\Omega), u_{0} \in H_{0}^{1}(\Omega) \cap H^{2}(\Omega)$ and the compatibility condition

$$
-\mu \Delta u_{0}+\nabla \pi\left(\rho_{0}\right)=\rho_{0}^{1 / 2} g, \quad \text { for some } g \in L^{2}(\Omega)
$$

is satisfied, then there exists a unique local strong solution $(\rho, u)$ to the initial boundary value problem. Independently of their work, H. J. Choe and H. Kim in [12, 13] proved a similar existence result when $\Omega$ is either a bounded domain or the whole space and the compatibility condition (1.4) is satisfied.

However, for the non-Newtonian fluid with initial vacuums, there was no existence result for strong solutions. This is somewhat surprising because in the context of the compressible Euler equations, there have been many works concerning the existence of a strong solution with compactly supported initial data. For these results, see [14, 15, 16].

Definition 1.1. The pair $(\rho, u)$ is called a strong solution to the initial boundary value problem (1.1)-(1.2), if the following conditions are satisfied:

(i)

$$
\begin{aligned}
& 0 \leqslant \rho \in C\left([0, T] ; H^{1}(I)\right), \quad \rho_{t} \in C\left([0, T] ; L^{2}(I)\right), \quad u_{t} \in L^{2}\left(0, T ; H_{0}^{1}(I)\right), \\
& u \in C\left([0, T] ; H_{0}^{1}(I)\right) \cap L^{\infty}\left(0, T ; H^{2}(I)\right), \quad \sqrt{\rho} u_{t} \in L^{\infty}\left(0, T ; L^{2}(I)\right) .
\end{aligned}
$$

(ii) For all $\varphi \in C\left([0, T] ; H^{1}(I)\right), \varphi_{t} \in L^{\infty}\left(0, T ; L^{2}(I)\right)$, for a.e. $t \in(0, T)$, we have:

$$
\int_{0}^{1} \rho(x, t) \varphi(x, t) \mathrm{d} x-\int_{0}^{t} \int_{0}^{1}\left(\rho \varphi_{t}+\rho u \varphi_{x}\right) \mathrm{d} x \mathrm{~d} s=\int_{0}^{1} \rho_{0} \varphi(x, 0) \mathrm{d} x .
$$


(iii) For all $\phi \in C\left([0, T] ; H_{0}^{1}(I)\right) \cap L^{\infty}\left(0, T ; H^{2}(I)\right), \phi_{t} \in L^{2}\left(0, T ; H_{0}^{1}(I)\right)$, for a.e. $t \in(0, T)$, we have:

$$
\begin{aligned}
& \int_{0}^{1} \rho u \phi(x, t) \mathrm{d} x-\int_{0}^{t} \int_{0}^{1}\left\{\rho u \phi_{t}+\rho u^{2} \phi_{x}+\pi \phi_{x}-\left[\left|u_{x}\right|^{2}+\mu_{0}\right]^{\frac{p-2}{2}} u_{x} \phi_{x}\right\}(x, s) \mathrm{d} x \mathrm{~d} s \\
& =\int_{0}^{1} \rho_{0} u_{0} \phi(x, 0) \mathrm{d} x+\int_{0}^{t} \int_{0}^{1} \rho f \phi(x, s) \mathrm{d} x \mathrm{~d} s .
\end{aligned}
$$

Now we can state the main result of this paper.

TheOrem 1.2. Assume that $\rho_{0}, u_{0}, f$ satisfy the following conditions:

$0 \leqslant \rho_{0} \in H^{1}(I), \quad u_{0} \in H_{0}^{1}(I) \cap H^{2}(I), \quad f \in L^{\infty}\left(0, T ; L^{2}(I)\right), \quad f_{t} \in L^{\infty}\left(0, T ; L^{2}(I)\right)$,

and if there is a function $g \in L^{2}(I)$, such that the following identity holds:

$$
-\left[\left(\left|u_{0 x}\right|^{2}+\mu_{0}\right)^{\frac{p-2}{2}} u_{0 x}\right]_{x}+\pi_{x}\left(\rho_{0}\right)=\rho_{0}^{\frac{1}{2}} g \quad \text { for a.e. } x \in I .
$$

Then there exists a $T_{*} \in(0,+\infty)$, such that the initial and boundary problem (1.1)-(1.2) has a unique strong solution $(\rho, u)$ in $\Omega_{T_{*}}$, satisfying the following properties:

$$
\left\{\begin{array}{l}
\rho \in C\left(\left[0, T_{*}\right] ; H^{1}(I)\right), \quad \rho_{t} \in C\left(\left[0, T_{*}\right] ; L^{2}(I)\right), \quad \sqrt{\rho} u_{t} \in L^{\infty}\left(0, T_{*} ; L^{2}(I)\right), \\
u \in C\left(\left[0, T_{*}\right] ; H_{0}^{1}(I)\right) \cap L^{\infty}\left(0, T_{*} ; H^{2}(I)\right), \quad u_{t} \in L^{2}\left(0, T_{*} ; H_{0}^{1}(I)\right), \\
\left(\left(u_{x}^{2}+\mu_{0}\right)^{\frac{p-2}{2}} u_{x}\right)_{x} \in C\left(\left[0, T_{*}\right] ; L^{2}(I)\right) .
\end{array}\right.
$$

The rest of this paper is organized as follows. In section 2, we first construct approximate solutions to the initial boundary value problem and obtain the uniform estimate on the approximate solutions. Finally, we obtain existence and uniqueness of solutions for the problem with positive densities. In section 3, we use the result of section 2 , and finish the proof of the main Theorem 1.2.

2. Local existence and uniqueness for positive densities. In this section, we prove local existence and uniqueness of solutions to the original problem (1.1)-(1.2) with positive densities. Furthermore, we derive some uniform bounds that are independent of the lower bounds of the initial density. The bounds will be used in the next section to prove the existence of strong solutions with nonnegative densities. We state the following proposition:

Proposition 2.1. Assume that $\rho_{0}, f$ are both sufficiently smooth functions, $\rho_{0} \geqslant \delta$ for some given constant $\delta>0$, and $f(0, t)=f(1, t)=0, u_{0} \in H_{0}^{1}(I) \cap H^{2}(I)$. If there exists some $g(x) \in L^{2}(I), g(0)=g(1)=0$, such that

$$
-\left[\left(u_{0 x}^{2}+\mu_{0}\right)^{\frac{p-2}{2}} u_{0 x}\right]_{x}+\pi_{x}\left(\rho_{0}\right)=\rho_{0}^{\frac{1}{2}} g,
$$


then there exists a $T_{*} \in(0,+\infty)$ such that the initial and boundary problem (1.1)-(1.2) has a unique strong solution $(\rho, u)$ in $\Omega_{T_{*}}$, satisfying the following properties:

$$
\left\{\begin{array}{l}
\rho \in C\left(\left[0, T_{*}\right] ; H^{1}(I)\right), \quad u \in C\left(\left[0, T_{*}\right] ; H_{0}^{1}(I)\right) \cap L^{\infty}\left(0, T_{*} ; H^{2}(I)\right), \\
\rho_{t} \in C\left(\left[0, T_{*}\right] ; L^{2}(I)\right), \quad u_{t} \in L^{2}\left(0, T_{*} ; H_{0}^{1}(I)\right), \quad \sqrt{\rho} u_{t} \in L^{\infty}\left(0, T_{*} ; L^{2}(I)\right), \\
\left(\left(u_{x}^{2}+\mu_{0}\right)^{\frac{p-2}{2}} u_{x}\right)_{x} \in C\left(\left[0, T_{*}\right] ; L^{2}(I)\right) .
\end{array}\right.
$$

2.1. Construction and uniform estimates of approximate solutions. Before constructing approximate solutions, we first prove the following lemma.

LEmma 2.2 (Embedding inequality). Assume that $f=0$ on $\partial \Omega$; here $\Omega \in \mathbb{R}^{1}$ is bounded and open, $f \in C^{2+\alpha}(\bar{\Omega})$. Then

$$
\left|f^{\prime}\right|_{L^{\infty}(\Omega)} \leqslant d^{\frac{1}{2}}(\Omega)\left|f^{\prime \prime}\right|_{L^{2}(\Omega)},
$$

where $d(\Omega)$ denotes the length of $\Omega$.

Proof. Since $f^{\prime}(y)$ can be divided into

$$
f^{\prime}(y)=f^{\prime}(\xi)+\int_{\xi}^{y} f^{\prime \prime}(\lambda) \mathrm{d} \lambda
$$

and $f \in C^{2+\alpha}(\bar{\Omega})$, then $f$ attains its maximum (minimum) on $\bar{\Omega}$.

(i) If there exists a point $\xi$ in $\Omega$ such that $f(\xi)$ attains its maximum (minimum), then $f^{\prime}(\xi)=0$.

(ii) If $f$ can't attain the maximum (minimum) in $\Omega$, then it attains either on boundary, and $f=0$ on $\partial \Omega$, thus $f \equiv 0$.

Therefore, if we can find a point $\xi$ in $\bar{\Omega}$ such that $f^{\prime}(\xi)=0$, then (2.9) may be rewritten $\forall y \in \bar{\Omega}$ as

$$
\begin{aligned}
f^{\prime}(y) & =\int_{\xi}^{y} f^{\prime \prime}(\lambda) \mathrm{d} \lambda \\
& \leqslant\left(\int_{\xi}^{y}\left(f^{\prime \prime}(\lambda)\right)^{2} \mathrm{~d} \lambda\right)^{\frac{1}{2}} \cdot(y-\xi)^{\frac{1}{2}} \\
& \leqslant d^{\frac{1}{2}}(\Omega)\left(\int_{\Omega}\left(f^{\prime \prime}(\lambda)\right)^{2} \mathrm{~d} \lambda\right)^{\frac{1}{2}} \\
& =d^{\frac{1}{2}}(\Omega)\left|f^{\prime \prime}(y)\right|_{L^{2}(\Omega)},
\end{aligned}
$$

where $d(\Omega)$ denotes the length of $\Omega$. Thus we have

$$
\left|f^{\prime}\right|_{L^{\infty}(\Omega)} \leqslant d^{\frac{1}{2}}(\Omega)\left|f^{\prime \prime}\right|_{L^{2}(\Omega)} .
$$

Now, we directly construct approximate solutions of (1.1)-(1.2) as follows.

Our first step is to define $u^{0}=0$ and substitute it into the following initial problem:

$$
\left\{\begin{array}{l}
\rho_{t}^{1}+u^{0} \rho_{x}^{1}+u_{x}^{0} \rho^{1}=0 \\
\rho^{1}(x, 0)=\rho_{0} .
\end{array}\right.
$$


Obviously, the initial value problem yields a unique smooth solution $\rho^{1}$.

Our second step is to substitute $\rho^{1}$ into the following initial boundary value problem:

$$
\left\{\begin{array}{l}
\rho^{1} u_{t}^{1}+\rho^{1} u^{0} u_{x}^{1}-\left[\left(\left(u_{x}^{1}\right)^{2}+\mu_{0}\right)^{\frac{p-2}{2}} u_{x}^{1}\right]_{x}+\pi_{x}^{1}=\rho^{1} f \quad(x, t) \in \Omega_{T}, \\
u^{1}(0, t)=u^{1}(1, t)=0, \\
u^{1}(x, 0)=u_{0} .
\end{array}\right.
$$

According to the classical existence theorem (see [17]), this initial boundary value problem yields a unique smooth solution $u^{1}$.

In the third step, we substitute $u^{1}$ into the following initial problem:

$$
\left\{\begin{array}{l}
\rho_{t}^{2}+u^{1} \rho_{x}^{2}+u_{x}^{1} \rho^{2}=0 \\
\rho^{2}(x, 0)=\rho_{0}
\end{array}\right.
$$

Using the existence theorem of the initial problem of the first-order linear hyperbolic equation, we can gain a unique smooth solution $\rho^{2}$ and substitute $\rho^{2}$ into the following initial boundary value problem:

$$
\left\{\begin{array}{l}
\rho^{2} u_{t}^{2}+\rho^{2} u^{1} u_{x}^{2}-\left[\left(\left(u_{x}^{2}\right)^{2}+\mu_{0}\right)^{\frac{p-2}{2}} u_{x}^{2}\right]_{x}+\pi_{x}^{2}=\rho^{2} f \quad(x, t) \in \Omega_{T}, \\
u^{2}(0, t)=u^{2}(1, t)=0, \\
u^{2}(x, 0)=u_{0} .
\end{array}\right.
$$

Then we have a smooth solution $u^{2}$. Using step-by-step iteration, we can thus obtain a number of smooth function sequences $\left\{\left(\rho^{k}, u^{k}\right)\right\}$, and $\left(\rho^{k}, u^{k}\right)$ is a unique smooth solution of the following initial boundary value problem:

$$
\begin{aligned}
& \rho_{t}^{k}+u^{k-1} \rho_{x}^{k}+u_{x}^{k-1} \rho^{k}=0, \\
& \rho^{k} u_{t}^{k}+\rho^{k} u^{k-1} u_{x}^{k}+\mathrm{E}_{p} u^{k}+\pi_{x}^{k}=\rho^{k} f, \quad(x, t) \in \Omega_{T}, \\
& \left.\rho^{k}\right|_{t=0}=\rho_{0},\left.\quad u^{k}\right|_{t=0}=u_{0}, \quad x \in \bar{I} ; \quad u^{k}(0, t)=u^{k}(1, t)=0, \quad t \in[0, T],
\end{aligned}
$$

where $\pi^{k} \equiv \pi\left(\rho^{k}\right)=A\left(\rho^{k}\right)^{\gamma}, A>0, \gamma>1$,

$$
\mathrm{E}_{p} u^{k}=-\left[\left(\left(u_{x}^{k}\right)^{2}+\mu_{0}\right)^{\frac{p-2}{2}} u_{x}^{k}\right]_{x},
$$

and $u_{0} \in H_{0}^{1}(I) \cap H^{2}(I)$ is a smooth solution of the following boundary value problem:

$$
\left\{\begin{array}{l}
{\left[\left(\left(u_{0 x}\right)^{2}+\mu_{0}\right)^{\frac{p-2}{2}} u_{0 x}\right]_{x}=\pi_{x}\left(\rho_{0}\right)-\rho_{0}^{\frac{1}{2}} g,} \\
u_{0}(0)=u_{0}(1)=0 .
\end{array}\right.
$$

Furthermore, by (2.13), we have

$$
\begin{aligned}
& \left(u_{0 x}^{2}+\mu_{0}\right)^{\frac{p-4}{2}}\left((p-1) u_{0 x}^{2}+\mu_{0}\right)\left|u_{0 x x}\right| \\
\geqslant & \left(u_{0 x}^{2}+\mu_{0}\right)^{\frac{p-4}{2}}\left(u_{0 x}^{2}+\mu_{0}\right)\left|u_{0 x x}\right| \\
= & \left(u_{0 x}^{2}+\mu_{0}\right)^{\frac{p-2}{2}}\left|u_{0 x x}\right| \\
\geqslant & \mu_{0}^{\frac{p-2}{2}}\left|u_{0 x x}\right| .
\end{aligned}
$$


Then

$$
\begin{aligned}
\left|u_{0 x x}\right|_{L^{2}(I)} & \leqslant \mu_{0}^{\frac{2-p}{2}}\left|\pi_{x}\left(\rho_{0}\right)-\rho_{0}^{\frac{1}{2}} g\right|_{L^{2}(I)} \\
& \leqslant \mu_{0}^{\frac{2-p}{2}}\left(A \gamma\left|\rho_{0 x}\right|_{L^{2}(I)}\left|\rho_{0}\right|_{L^{\infty}(I)}^{\gamma-1}+\left|\rho_{0}\right|_{L^{\infty}(I)}^{\frac{1}{2}}|g|_{L^{2}(I)}\right) \\
& \leqslant C
\end{aligned}
$$

where $C$ is a positive constant, depending only on $M^{0}$. Throughout the paper, we denote by

$$
M^{0}=1+\mu_{0}+\mu_{0}^{-1}+\left|\rho_{0}\right|_{H^{1}(I)}+|g|_{L^{2}(I)}+|f|_{L^{\infty}\left(0, T ; L^{2}(I)\right)}+\left|f_{t}\right|_{L^{\infty}\left(0, T ; L^{2}(I)\right)} .
$$

From now on, we derive uniform bounds on the approximate solutions, including $\left|u_{x x}^{k}\right|_{L^{2}(I)}$.

Let $K \geqslant 1$ be a fixed integer, and let us construct an auxiliary function

$$
\Phi_{K}(t)=\max _{1 \leqslant k \leqslant K} \sup _{0 \leqslant s \leqslant t}\left(1+\left|\rho^{k}(s)\right|_{H^{1}(I)}+\left|u^{k}(s)\right|_{W_{0}^{1, p}(I)}+\left|\sqrt{\rho^{k}} u_{t}^{k}(s)\right|_{L^{2}(I)}\right) .
$$

First, we estimate $\left|u^{k}(s)\right|_{W_{0}^{1, p}(I)}$ of $\Phi_{K}(t)$.

Multiplying (2.11) by $u_{t}^{k}$ and integrating it over $(0,1)$ on $x$, we deduce that

$$
\begin{aligned}
& \int_{0}^{1} \rho^{k}\left|u^{k}\right|^{2} \mathrm{~d} x+\int_{0}^{1}\left[\left(\left(u_{x}^{k}\right)^{2}+\mu_{0}\right)^{\frac{p-2}{2}} u_{x}^{k}\right] u_{x t}^{k} \mathrm{~d} x \\
= & \frac{\mathrm{d}}{\mathrm{d} t} \int_{0}^{1} \pi^{k} u_{x}^{k} \mathrm{~d} x+\int_{0}^{1}\left[\left(\rho f-\rho u^{k-1} u_{x}^{k}\right) u_{t}^{k}-\pi_{t}^{k} u_{x}^{k}\right] \mathrm{d} x,
\end{aligned}
$$

and integrating over $(0, t)$ on the time variable for (2.15), we have

$$
\begin{aligned}
& \int_{0}^{t} \int_{0}^{1} \rho^{k}\left|u_{t}^{k}\right|^{2} \mathrm{~d} x \mathrm{~d} s+\int_{0}^{t} \int_{0}^{1}\left(\left(u_{x}^{k}\right)^{2}+\mu_{0}\right)^{\frac{p-2}{2}} u_{x}^{k} u_{x t}^{k} \mathrm{~d} x \mathrm{~d} s \\
= & -\int_{0}^{1} \pi^{k} u_{x}^{k}(0) \mathrm{d} x+\int_{0}^{1} \pi^{k} u_{x}^{k}(t) \mathrm{d} x+\int_{0}^{t} \int_{0}^{1}\left[\left(\rho f-\rho u^{k-1} u_{x}^{k}\right) u_{t}^{k}-\pi_{t}^{k} u_{x}^{k}\right] \mathrm{d} x \mathrm{~d} s .
\end{aligned}
$$

We first compute the second term of (2.16) to get

$$
\begin{aligned}
\int_{0}^{1}\left(\left(u_{x}^{k}\right)^{2}+\mu_{0}\right)^{\frac{p-2}{2}} u_{x}^{k} u_{x t}^{k} \mathrm{~d} x & =\frac{1}{2} \int_{0}^{1}\left(\left(u_{x}^{k}\right)^{2}+\mu_{0}\right)^{\frac{p-2}{2}}\left(u_{x}^{k}\right)_{t}^{2} \mathrm{~d} x \\
& =\frac{1}{2} \frac{\mathrm{d}}{\mathrm{d} t} \int_{0}^{1}\left(\int_{0}^{\left(u_{x}^{k}\right)^{2}}\left(s^{2}+\mu_{0}\right)^{\frac{p-2}{2}} \mathrm{~d} s\right) \mathrm{d} x,
\end{aligned}
$$


and

$$
\begin{aligned}
& \int_{0}^{\left(u_{x}^{k}\right)^{2}}\left(s+\mu_{0}\right)^{\frac{p-2}{2}} \mathrm{~d} s \\
= & \int_{\mu_{0}}^{\left(u_{x}^{k}\right)^{2}+\mu_{0}} t^{\frac{p-2}{2}} \mathrm{~d} t \\
= & \frac{2}{p}\left[\left(\left(u_{x}^{k}\right)^{2}+\mu_{0}\right)^{\frac{p}{2}}-\mu_{0}^{\frac{p}{2}}\right] \\
\geqslant & \frac{2}{p}\left|u_{x}^{k}\right|^{p}-\frac{2}{p} \mu_{0}^{\frac{p}{2}} .
\end{aligned}
$$

Substituting (2.17), (2.18) into (2.16), we have

$$
\begin{aligned}
& \int_{0}^{t}\left|\sqrt{\rho^{k}} u_{t}^{k}(s)\right|_{L^{2}(I)}^{2} \mathrm{~d} s+\frac{1}{p} \int_{0}^{1}\left|u_{x}^{k}(t)\right|^{p} \mathrm{~d} x \\
\leqslant & \frac{2}{p}\left|u_{x}^{k}(0)\right|_{L^{p}(I)}^{p}+\left|\pi^{k}(0)\right|_{L^{2}(I)}^{2}+\frac{2}{p} \mu_{0}^{\frac{p}{2}}+\int_{0}^{1}\left|\pi^{k} u_{x}^{k}(t)\right| \mathrm{d} x \\
& +\int_{0}^{t} \int_{0}^{1}\left|\left(\rho^{k} f-\rho^{k} u^{k-1} u_{x}^{k}\right) u_{t}^{k}-\pi_{t}^{k} u_{x}^{k}\right| \mathrm{d} x \mathrm{~d} s \\
\leqslant & C+\int_{0}^{1}\left|\pi^{k} u_{x}^{k}(t)\right| \mathrm{d} x+\int_{0}^{t} \int_{0}^{1}\left|\left(\rho^{k} f-\rho^{k} u^{k-1} u_{x}^{k}\right) u_{t}^{k}-\pi_{t}^{k} u_{x}^{k}\right| \mathrm{d} x \mathrm{~d} s,
\end{aligned}
$$

where $C$ is a positive constant, depending only on $M_{0}$.

By (2.10), we have

$$
\pi_{t}^{k}=-\gamma \pi^{k} u_{x}^{k-1}-\pi_{x}^{k} u^{k-1}
$$


Substituting it into (2.19), we have

$$
\begin{aligned}
& \int_{0}^{t}\left|\sqrt{\rho^{k}} u_{t}^{k}(s)\right|_{L^{2}(I)}^{2} \mathrm{~d} s+\left|u_{x}^{k}(t)\right|_{L^{p}}^{p} \\
& \leqslant C+\int_{0}^{t} \int_{0}^{1}\left(\left|\rho^{k} f u_{t}^{k}\right|+\left|\rho^{k} u^{k-1} u_{x}^{k} u_{t}^{k}\right|+\left|\pi_{x}^{k} u^{k-1} u_{x}^{k}\right|+\gamma\left|\pi u_{x}^{k-1} u_{x}^{k}\right|\right) \mathrm{d} x \mathrm{~d} s \\
& +C\left|\pi^{k}\right|_{L^{2}(I)}^{\frac{p}{p-1}}+\frac{1}{2}\left|u_{x}^{k}(t)\right|_{L^{p}(I)}^{p} \\
& \leqslant C+\eta \int_{0}^{t}\left|\sqrt{\rho^{k}} u_{x}^{k}(s)\right|_{L^{2}(I)}^{2} \mathrm{~d} s+C_{\eta} \int_{0}^{t}\left|\sqrt{\rho^{k}} f(s)\right|_{L^{2}(I)}^{2} \mathrm{~d} s \\
& +C_{\eta} \int_{0}^{t}\left|\rho^{k}(s)\right|_{L^{\infty}(I)}\left|u^{k-1}(s)\right|_{L^{\infty}(I)}^{2}\left|u_{x}^{k}(s)\right|_{L^{p}(I)}^{2} \mathrm{~d} s \\
& +\int_{0}^{t}\left(\left|\pi_{x}^{k}(s)\right|_{L^{2}(I)}\left|u^{k-1}(s)\right|_{L^{\infty}(I)}\left|u_{x}^{k}(s)\right|_{L^{p}(I)}\right. \\
& \left.+\gamma|\pi(s)|_{L^{\infty}(I)}\left|u_{x}^{k-1}(s)\right|_{L^{p}(I)}\left|u_{x}^{k}(s)\right|_{L^{p}(I)}\right) \mathrm{d} s \\
& +C\left|\pi^{k}(t)\right|_{L^{2}(I)}^{\frac{p}{p-1}}+\frac{1}{2}\left|u_{x}^{k}(t)\right|_{L^{p}(I)}^{p},
\end{aligned}
$$

where $0<\eta<<1$. The above inequality can be written as:

$$
\begin{aligned}
& \int_{0}^{t}\left|\sqrt{\rho^{k}} u_{t}^{k}(s)\right|_{L^{2}(I)}^{2} \mathrm{~d} s+\left|u_{x}^{k}(t)\right|_{L^{p}(I)}^{p} \\
\leqslant & C+\int_{0}^{t}\left(\left|\sqrt{\rho^{k}} f(s)\right|_{L^{2}(I)}^{2}+\left|\rho^{k}(s)\right|_{L^{\infty}(I)}\left|u_{x}^{k-1}(s)\right|_{L^{p}(I)}^{2}\left|u_{x}^{k}(s)\right|_{L^{p}(I)}^{2}\right) \mathrm{d} s \\
& +\int_{0}^{t}\left|\pi_{x}^{k}(s)\right|_{L^{2}(I)}\left|u_{x}^{k-1}(s)\right|_{L^{p}(I)}\left|u_{x}^{k}(s)\right|_{L^{p}(I)} \mathrm{d} s+C\left|\pi^{k}(t)\right|_{L^{2}(I)}^{2} \\
\leqslant & C \int_{0}^{t} \Phi_{K}^{\gamma+4}(s) \mathrm{d} s+C\left|\pi^{k}(t)\right|_{L^{2}(I)}^{2} .
\end{aligned}
$$

To estimate the right of (2.20), we can first obtain

$$
\begin{aligned}
& \left|\rho^{k}(t)\right|_{L^{\infty}(I)}+\left|\pi^{k}(t)\right|_{H^{1}(I)} \\
\leqslant & \left|\rho^{k}(t)\right|_{H^{1}(I)}+A\left|\rho^{k}(t)\right|_{L^{\infty}(I)}^{\gamma-1}\left|\rho^{k}(t)\right|_{H^{1}(I)} \\
\leqslant & C \Phi_{K}^{\gamma}(t) .
\end{aligned}
$$


By (2.10), we have

$$
\begin{aligned}
& \int_{0}^{1}\left|\pi^{k}(t)\right|^{2} \mathrm{~d} x \\
= & \int_{0}^{1}\left|\pi^{k}(0)\right|^{2} \mathrm{~d} x+\int_{0}^{t} \frac{\partial}{\partial s}\left(\int_{0}^{1}\left(\pi^{k}(s)\right)^{2} \mathrm{~d} x\right) \mathrm{d} s \\
= & \int_{0}^{1}\left|\pi^{k}(0)\right|^{2} \mathrm{~d} x+2 \int_{0}^{t} \int_{0}^{1}\left(\pi^{k}\left(\rho^{k}\right)\right)^{\prime} \pi^{k}\left(-\rho_{x}^{k} u^{k-1}-\rho^{k} u_{x}^{k-1}\right) \mathrm{d} x \mathrm{~d} s \\
\leqslant & C+C \int_{0}^{t}\left|\rho^{k}(s)\right|_{L^{\infty}(I)}^{\gamma-1}\left|\pi^{k}(s)\right|_{L^{\infty}(I)}\left|\rho_{x}^{k}(s)\right|_{L^{2}(I)}\left|u_{x}^{k-1}(s)\right|_{L^{p}(I)} \mathrm{d} s \\
\leqslant & C\left(1+\int_{0}^{t} \Phi_{K}^{\gamma+2}(s) \mathrm{d} s\right),
\end{aligned}
$$

where $C$ is a positive constant, depending only on $M^{0}$. Substituting it into (2.20), we have

$$
\int_{0}^{t}\left|\sqrt{\rho^{k}} u_{t}^{k}(s)\right|_{L^{2}(I)}^{2} \mathrm{~d} s+\left|u_{x}^{k}(t)\right|_{L^{p}(I)}^{p} \leqslant C\left(1+\int_{0}^{t} \Phi_{K}^{\gamma+4}(s) \mathrm{d} s\right)
$$

for all $k, 1 \leqslant k \leqslant K$, where $C$ is a positive constant, depending only on $M^{0}$.

Second, we will estimate the $\left|\sqrt{\rho^{k}} u_{t}^{k}(t)\right|_{L^{2}(I)}$ of $\Phi_{K}(t)$.

We first estimate $\left|u_{x x}(t)\right|_{L^{2}(I)}$. By (2.11), we have

$$
\left[\left(\left(u_{x}^{k}\right)^{2}+\mu_{0}\right)^{\frac{p-2}{2}} u_{x}^{k}\right]_{x}=\rho^{k} u_{t}^{k}+\rho u^{k-1} u_{x}^{k}+\pi_{x}^{k}-\rho^{k} f .
$$

Since

$$
\left|\left(\left(\left(u_{x}^{k}\right)^{2}+\mu_{0}\right)^{\frac{p-2}{2}} u_{x}^{k}\right)_{x}\right| \geqslant \mu_{0}^{\frac{p-2}{2}}\left|u_{x x}\right|
$$

then

$$
\left|u_{x x}^{k}\right| \leqslant C\left|\rho^{k} u_{t}^{k}+\rho^{k} u^{k-1} u_{x}^{k}+\pi_{x}^{k}-\rho^{k} f\right| .
$$

Taking it by the $L^{2}$ norm, the inequality still holds:

$$
\begin{aligned}
& \left|u_{x x}^{k}(t)\right|_{L^{2}(I)} \\
\leqslant & C\left|\rho^{k} u_{t}^{k}+\rho^{k} u^{k-1} u_{x}^{k}+\pi_{x}^{k}-\rho^{k} f\right|_{L^{2}(I)} \\
\leqslant & C\left(\left|\rho^{k} u_{t}^{k}(t)\right|_{L^{2}(I)}+\left|\rho^{k} u^{k-1} u_{x}^{k}(t)\right|_{L^{2}(I)}+\left|\pi_{x}^{k}(t)\right|_{L^{2}(I)}+\left|\rho^{k} f(t)\right|_{L^{2}(I)}\right) \\
\leqslant & C\left(\left|\rho^{k}\right|_{L^{\infty}(I)}^{\frac{1}{2}}\left|\sqrt{\rho^{k}} u_{t}^{k}(t)\right|_{L^{2}(I)}+\left|\rho^{k}\right|_{L^{\infty}(I)}\left|u^{k-1}\right|_{L^{\infty}(I)}\left|u_{x}^{k}(t)\right|_{L^{p}(I)}\right. \\
& \left.+\left|\pi_{x}^{k}(t)\right|_{L^{2}(I)}+\left|\rho^{k} f(t)\right|_{L^{2}(I)}\right) .
\end{aligned}
$$


Hence, we deduce that

$$
\left|u_{x x}^{k}(t)\right|_{L^{2}(I)} \leqslant C \Phi_{K}^{\gamma+2}(t)
$$

We differentiate (2.11) with respect to $t$ and multiply it by $u_{t}^{k}$, and by integrating it over $(0,1)$ on $x$, we have

$$
\begin{aligned}
& \frac{1}{2} \frac{\mathrm{d}}{\mathrm{d} t} \int_{0}^{1} \rho^{k}\left|u_{t}^{k}\right|^{2}(t) \mathrm{d} x+\int_{0}^{1}\left[\left(\left(u_{x}^{k}\right)^{2}+\mu_{0}\right)^{\frac{p-2}{2}} u_{x}^{k}\right]_{t} u_{x t}^{k}(t) \mathrm{d} x \\
= & \int_{0}^{1}\left[\left(\rho^{k} u^{k-1}\right)_{x}\left(u_{t}^{k}+u^{k-1} u_{x}^{k}-f\right)-\rho^{k} u_{t}^{k-1} u_{x}^{k}+\rho^{k} f_{t}\right] u_{t}^{k} \mathrm{~d} x .
\end{aligned}
$$

Since

$$
\begin{aligned}
& {\left[\left(\left(u_{x}^{k}\right)^{2}+\mu_{0}\right)^{\frac{p-2}{2}} u_{x}^{k}\right]_{t} u_{x t}^{k} } \\
= & \left(\left(u_{x}^{k}\right)^{2}+\mu_{0}\right)^{\frac{p-4}{2}}\left((p-1)\left(u_{x}^{k}\right)^{2}+\mu_{0}\right)\left(u_{x t}^{k}\right)^{2} \\
\geqslant & \mu_{0}^{\frac{p-2}{2}}\left(u_{x t}^{k}\right)^{2}
\end{aligned}
$$

then (2.23) may be written as:

$$
\begin{aligned}
& \frac{\mathrm{d}}{\mathrm{d} t} \int_{0}^{1} \rho^{k}\left|u_{t}^{k}\right|(t) \mathrm{d} x+\int_{0}^{1}\left|u_{x t}^{k}\right|^{2} \mathrm{~d} x \\
\leqslant & C \int_{0}^{1} \pi_{t}^{k} u_{x t}^{k} \mathrm{~d} x+\int_{0}^{1}\left[\left(\rho^{k} u^{k-1}\right)_{x}\left(u_{t}^{k}+u^{k-1} u_{x}^{k}-f\right)-\rho^{k} u_{t}^{k-1} u_{x}^{k}+\rho^{k} f_{t}\right] u_{t}^{k} \mathrm{~d} x \\
\leqslant & C \int_{0}^{1}\left(2 \rho^{k}\left|u^{k-1}\right|\left|u_{t}^{k}\right|\left|u_{x t}^{k}\right|+\rho^{k}\left|u^{k-1}\right|\left|u_{x}^{k-1}\right|\left|u_{x}^{k}\right|\left|u_{t}^{k}\right|+\left|\rho_{x}^{k}\right|\left|u^{k-1}\right|^{2}\left|u_{t}^{k}\right|\left|u_{x}^{k}\right|\right. \\
& +\rho^{k}\left|u_{t}^{k-1}\right|\left|u_{t}^{k}\right|\left|u_{x}^{k}\right|+\left|\pi_{x}^{k}\right|\left|u^{k-1}\right|\left|u_{x t}^{k}\right|+\gamma \pi^{k}\left|u_{x}^{k-1}\right|\left|u_{x t}^{k}\right|+\left|\rho_{x}^{k}\right|\left|u^{k-1}\right||f|\left|u_{t}^{k}\right| \\
& \left.+\rho^{k}\left|u_{x}^{k-1}\right||f|\left|u_{t}^{k}\right|+\rho^{k}\left|u^{k-1}\right||f|\left|u_{x t}^{k}\right|+\rho^{k}\left|u_{t}^{k}\right|\left|f_{t}\right|\right) \mathrm{d} x \\
= & C \sum_{j=1}^{10} I_{j} .
\end{aligned}
$$


By Sobolev inequality and Young's inequality, we obtain

$$
\begin{aligned}
& I_{1}=\int_{0}^{1} 2 \rho^{k}\left|u^{k-1}\right|\left|u_{t}^{k}\right|\left|u_{x t}^{k}\right| \mathrm{d} x \\
& \leqslant 2\left|\rho^{k}\right|_{L^{\infty}(I)}^{\frac{1}{2}}\left|u^{k-1}\right|_{L^{\infty}(I)}\left|\sqrt{\rho^{k}} u_{t}^{k}\right|_{L^{2}(I)}\left|u_{x t}^{k}(t)\right|_{L^{2}(I)} \\
& \leqslant C \Phi_{K}^{5}(t)+\frac{1}{8}\left|u_{x t}^{k}\right|_{L^{2}(I)}^{2}, \\
& I_{2}=\int_{0}^{1} \rho^{k}\left|u^{k-1}\right|\left|u_{x}^{k-1}\right|\left|u_{x}^{k}\right|\left|u_{t}^{k}\right| \mathrm{d} x \\
& \leqslant\left|\rho^{k}\right|_{L^{\infty}(I)}\left|u^{k-1}\right|_{L^{\infty}(I)}\left|u_{x}^{k-1}\right|_{L^{p}(I)}\left|u_{x}^{k}\right|_{L^{p}}\left|u_{t}^{k}\right|_{L^{\infty}(I)} \\
& \leqslant\left|\rho^{k}\right|_{L^{\infty}(I)}\left|u_{x}^{k-1}\right|_{L^{p}(I)}^{2}\left|u_{x}^{k}\right|_{L^{p}(I)}\left|u_{x t}^{k}(t)\right|_{L^{2}(I)} \\
& \leqslant C \Phi_{K}^{8}(t)+\frac{1}{8}\left|u_{x t}^{k}(t)\right|_{L^{2}(I)}^{2}, \\
& I_{3}=\int_{0}^{1}\left|\rho_{x}^{k}\right|\left|u^{k-1}\right|^{2}\left|u_{t}^{k}\right|\left|u_{x}^{k}\right| \mathrm{d} x \\
& \leqslant\left|\rho_{x}^{k}\right|_{L^{2}(I)}\left|u^{k-1}\right|_{L^{\infty}(I)}^{2}\left|u_{t}^{k}\right|_{L^{\infty}(I)}\left|u_{x}^{k}\right|_{L^{p}(I)} \\
& \leqslant\left|\rho_{x}^{k}\right|_{L^{2}(I)}\left|u_{x}^{k-1}\right|_{L^{p}(I)}^{2}\left|u_{x t}^{k}\right|_{L^{2}(I)}\left|u_{x}^{k}\right|_{L^{p}(I)} \\
& \leqslant C \Phi_{K}^{8}(t)+\frac{1}{8}\left|u_{x t}^{k}(t)\right|_{L^{2}(I)}^{2}, \\
& I_{4}=\int_{0}^{1} \rho^{k}\left|u_{t}^{k-1}\right|\left|u_{t}^{k}\right|\left|u_{x}^{k}\right| \mathrm{d} x \\
& \leqslant\left|\rho^{k}\right|_{L^{\infty}(I)}^{\frac{3}{4}}\left|u_{t}^{k-1}\right|_{L^{\infty}(I)}\left|\sqrt{\rho^{k}} u_{t}^{k}\right|_{L^{2}(I)}^{\frac{1}{2}}\left|u_{t}^{k}\right|_{L^{\infty}(I)}^{\frac{1}{2}}\left|u_{x}^{k}\right|_{L^{p}(I)} \\
& \leqslant\left|\rho^{k}\right|_{L^{\infty}(I)}^{\frac{3}{4}}\left|u_{x t}^{k-1}\right|_{L^{2}(I)}\left|u_{x t}^{k}\right|_{L^{2}(I)}^{\frac{1}{2}}\left|\sqrt{\rho^{k}} u_{t}^{k}\right|_{L^{2}(I)}^{\frac{1}{2}}\left|u_{x}^{k}\right|_{L^{p}(I)} \\
& \leqslant C \Phi_{K}^{\frac{9}{2}}(t)\left|u_{x t}^{k}\right|_{L^{2}(I)}+\frac{1}{2}\left|u_{x t}^{k-1}\right|_{L^{2}(I)}^{2} \\
& \leqslant C \Phi_{K}^{9}(t)+\frac{1}{2}\left|u_{x t}^{k-1}(t)\right|_{L^{2}(I)}^{2}+\frac{1}{8}\left|u_{x t}^{k}(t)\right|_{L^{2}(I)}^{2},
\end{aligned}
$$




$$
\begin{aligned}
& I_{5}=\int_{0}^{1}\left|\pi_{x}^{k}\right|\left|u^{k-1}\right|\left|u_{x t}^{k}\right| \mathrm{d} x \\
& \leqslant\left|\pi_{x}^{k}\right|_{L^{2}(I)}\left|u^{k-1}\right|_{L^{\infty}(I)}\left|u_{x t}^{k}\right|_{L^{2}(I)} \\
& \leqslant C \Phi_{K}^{2(\gamma+1)}(t)+\frac{1}{8}\left|u_{x t}^{k}\right|_{L^{2}(I)}^{2}, \\
& I_{6}=\int_{0}^{1} \gamma \pi^{k}\left|u_{x}^{k-1}\right|\left|u_{x t}^{k}\right| \mathrm{d} x \\
& \leqslant C\left|\pi^{k}\right|_{L^{\infty}(I)}\left|u_{x}^{k-1}\right|_{L^{p}(I)}\left|u_{x t}^{k}\right|_{L^{2}(I)} \\
& \leqslant C\left|\pi_{x}^{k}\right|_{L^{2}(I)}\left|u_{x}^{k-1}\right|_{L^{p}(I)}\left|u_{x t}^{k}\right|_{L^{2}(I)} \\
& \leqslant C \Phi_{K}^{2(\gamma+1)}(t)+\frac{1}{8}\left|u_{x t}^{k}(t)\right|_{L^{2}(I)}^{2}, \\
& I_{7}=\int_{0}^{1}\left|\rho_{x}^{k}\right|\left|u^{k-1}\right||f|\left|u_{t}^{k}\right| \mathrm{d} x \\
& \leqslant\left|\rho_{x}^{k}\right|_{L^{2}(I)}\left|u^{k-1}\right|_{L^{\infty}(I)}|f|_{L^{2}(I)}\left|u_{t}^{k}\right|_{L^{\infty}(I)} \\
& \leqslant\left|\rho_{x}^{k}\right|_{L^{2}(I)}\left|u_{x}^{k-1}\right|_{L^{p}(I)}|f|_{L^{2}(I)}\left|u_{x t}^{k}\right|_{L^{2}(I)} \\
& \leqslant C \Phi_{K}^{4}(t)+\frac{1}{8}\left|u_{x t}^{k}\right|_{L^{2}(I)}^{2}, \\
& I_{8}=\int_{0}^{1} \rho^{k}\left|u_{x}^{k-1}\right||f|\left|u_{t}^{k}\right| \mathrm{d} x \\
& \leqslant\left|\rho^{k}\right|_{L^{\infty}(I)}\left|u_{t}^{k}\right|_{L^{\infty}(I)}|f|_{L^{2}(I)}\left|u_{x}^{k-1}\right|_{L^{p}(I)} \\
& \leqslant C \Phi_{K}^{4}(t)+\frac{1}{8}\left|u_{x t}\right|_{L^{2}(I)}^{2}, \\
& I_{9}=\int_{0}^{1} \rho^{k}\left|u^{k-1}\right||f|\left|u_{x t}^{k}\right| \mathrm{d} x \\
& \leqslant\left|\rho^{k}\right|_{L^{\infty}(I)}\left|u^{k-1}\right|_{L^{\infty}(I)}|f|_{L^{2}(I)}\left|u_{x t}^{k}\right|_{L^{2}(I)} \\
& \leqslant\left|\rho^{k}\right|_{L^{\infty}(I)}\left|u_{x}^{k-1}\right|_{L^{p}(I)}|f|_{L^{2}(I)}\left|u_{x t}^{k}\right|_{L^{2}(I)} \\
& \leqslant C \Phi_{K}^{4}(t)+\frac{1}{8}\left|u_{x t}^{k}\right|_{L^{2}(I)}^{2},
\end{aligned}
$$




$$
\begin{aligned}
I_{10} & =\int_{0}^{1} \rho^{k}\left|u_{t}^{k}\right|\left|f_{t}\right| \mathrm{d} x \\
& \leqslant\left|\rho^{k}\right|_{L^{\infty}(I)}^{\frac{1}{2}}\left|\sqrt{\rho^{k}} u_{t}^{k}\right|_{L^{2}(I)}\left|f_{t}\right|_{L^{2}(I)} \\
& \leqslant C \Phi_{K}^{\frac{3}{2}}(t) .
\end{aligned}
$$

Substituting $I_{j}(j=1,2, \cdots, 10)$ into (2.24) and integrating it over $(\tau, t)$ on the time variable yields

$$
\begin{aligned}
& \left|\sqrt{\rho^{k}} u_{t}^{k}(t)\right|_{L^{2}(I)}^{2}+\int_{\tau}^{t}\left|u_{x t}^{k}\right|_{L^{2}}^{2}(s) \mathrm{d} s \\
\leqslant & C \int_{\tau}^{t} \Phi_{K}^{2 \gamma+7}(s) \mathrm{d} s+\left|\sqrt{\rho^{k}} u_{t}^{k}(\tau)\right|_{L^{2}(I)}^{2}+\frac{1}{2} \int_{\tau}^{t}\left|u_{x t}^{k-1}\right|_{L^{2}(I)}^{2}(s) \mathrm{d} s \\
\leqslant & C \int_{\tau}^{t} \Phi_{K}^{2 \gamma+7}(s) \mathrm{d} s+\sup _{1 \leqslant k \leqslant K}\left(1+\left|\sqrt{\rho^{k}} u_{t}^{k}(\tau)\right|_{L^{2}(I)}^{2}\right)+\frac{1}{2} \int_{\tau}^{t}\left|u_{x t}^{k-1}\right|_{L^{2}(I)}^{2}(s) \mathrm{d} s .
\end{aligned}
$$

From the above recursive relation, we obtain

$$
\begin{aligned}
& \int_{\tau}^{t}\left|u_{x t}^{k}\right|_{L^{2}(I)}^{2}(s) \mathrm{d} s \\
\leqslant & \left(1+\frac{1}{2}+\frac{1}{4}+\cdots \frac{1}{2^{K}}\right) C\left[\int_{\tau}^{t} \Phi_{K}^{2 \gamma+7}(s) \mathrm{d} s+\sup _{0 \leqslant k \leqslant K}\left(1+\left|\sqrt{\rho^{k}} u_{t}^{k}(\tau)\right|_{L^{2}(I)}^{2}\right)\right] \\
\leqslant & 2 C\left[\int_{\tau}^{t} \Phi_{K}^{2 \gamma+7}(s) \mathrm{d} s+\sup _{0 \leqslant k \leqslant K}\left(1+\left|\sqrt{\rho^{k}} u_{t}^{k}(\tau)\right|_{L^{2}(I)}^{2}\right)\right]
\end{aligned}
$$

for $1 \leqslant k \leqslant K$. According to the above inequality, (2.25) may be written as

$$
\begin{aligned}
& \left|\sqrt{\rho^{k}} u_{t}^{k}(t)\right|_{L^{2}(I)}^{2}+\int_{\tau}^{t}\left|u_{x t}^{k}\right|_{L^{2}}^{2}(s) \mathrm{d} s \\
\leqslant & C \int_{\tau}^{t} \Phi_{K}^{2 \gamma+7}(s) \mathrm{d} s+\sup _{0 \leqslant k \leqslant K}\left(1+\left|\sqrt{\rho^{k}} u_{t}^{k}(\tau)\right|_{L^{2}(I)}^{2}\right),
\end{aligned}
$$

where $C$ is a positive constant, depending only on $M^{0}$.

To obtain the estimate of $\left|\sqrt{\rho^{k}} u_{t}^{k}(t)\right|_{L^{2}(I)}^{2}$, we need to estimate

$$
\lim _{\tau \rightarrow 0} \sup _{0 \leqslant k \leqslant K}\left(1+\left|\sqrt{\rho^{k}} u_{t}^{k}(\tau)\right|_{L^{2}(I)}^{2}\right)
$$

By (2.11), we have

$$
\int_{0}^{1} \rho^{k}\left|u_{t}^{k}\right|^{2}(t) \mathrm{d} x \leqslant 2 \int_{0}^{1}\left(\rho^{k}\left|u^{k-1}\right|^{2}\left|u_{x}^{k}\right|^{2}+\rho^{k}|f|^{2}+\left(\rho^{k}\right)^{-1}\left|\mathrm{E}_{p} u^{k}+\pi_{x}^{k}\right|^{2}\right) \mathrm{d} x .
$$


According to the smoothing of $\left(\rho^{k}, u^{k}\right)$, we get

$$
\begin{aligned}
& \lim _{t \rightarrow 0} \int_{0}^{1}\left(\rho^{k}\left|u^{k-1}\right|^{2}\left|u_{x}^{k}\right|^{2}+\rho^{k}|f|^{2}+\left(\rho^{k}\right)^{-1}\left|\mathrm{E}_{p} u^{k}+\pi_{x}^{k}\right|^{2}\right)(x, t) \mathrm{d} x \\
= & \int_{0}^{1}\left(\rho_{0}\left|u_{0}\right|^{2}\left|u_{0 x}\right|^{2}+\rho_{0}|f|^{2}+\left(\rho_{0}\right)^{-1}\left|\mathrm{E}_{p} u_{0}+\pi_{x}^{k}\left(\rho_{0}\right)\right|^{2}\right)(x, t) \mathrm{d} x \\
= & \int_{0}^{1}\left(\rho_{0}\left|u_{0}\right|^{2}\left|u_{0 x}\right|^{2}+\rho_{0}|f|^{2}\right) \mathrm{d} x+|g|_{L^{2}(I)}^{2} \\
\leqslant & \left|\rho_{0}\right|_{L^{\infty}(I)}\left|u_{0}\right|_{L^{\infty}(I)}\left|u_{0 x}\right|_{L^{2}(I)}^{2}+\left|\rho_{0}\right|_{L^{\infty}(I)}|f|_{L^{2}(I)}+|g|_{L^{2}(I)}^{2} .
\end{aligned}
$$

By (2.14), we obtain that

$$
\lim _{\tau \rightarrow 0} \sup \int_{0}^{1} \rho^{k}\left|u_{t}^{k}\right|^{2}(\tau) \mathrm{d} x \leqslant C,
$$

where $C$ is a positive constant, depending only on $M^{0}$.

Taking (2.26) the limit on $\tau$, letting $\tau \rightarrow 0$, we have

$$
\left|\sqrt{\rho^{k}} u_{t}^{k}(t)\right|_{L^{2}(I)}^{2}+\int_{0}^{t}\left|u_{x t}^{k}\right|_{L^{2}}^{2}(s) \mathrm{d} s \leqslant C_{1}+C_{2} \int_{0}^{t} \Phi_{K}^{2 \gamma+7}(s) \mathrm{d} s .
$$

Multiplying (2.10) by $\rho^{k}$ and integrating over $(0,1)$ with respect to $x$, we have

$$
\frac{1}{2} \frac{\mathrm{d}}{\mathrm{d} t} \int_{0}^{1}\left|\rho^{k}(t)\right|^{2} \mathrm{~d} x+\int_{0}^{1}\left(\rho u^{k-1}\right)_{x} \rho^{k}(t) \mathrm{d} x=0 .
$$

Integrating by parts, we have

$$
\frac{1}{2} \frac{\mathrm{d}}{\mathrm{d} t} \int_{0}^{1}\left|\rho^{k}(t)\right|^{2} \mathrm{~d} x \leqslant \frac{1}{2} \int_{0}^{1}\left|u_{x}^{k-1}\right|\left|\rho^{k}\right|^{2}(t) \mathrm{d} x .
$$

Using Sobolev inequality, we have

$$
\frac{\mathrm{d}}{\mathrm{d} t}\left|\rho^{k}(t)\right|_{L^{2}(I)} \leqslant C\left|u_{x x}^{k-1}(t)\right|_{L^{2}(I)}\left|\rho^{k}(t)\right|_{L^{2}(I)} .
$$

Then differentiating (2.10) with respect to $x$, multiplying it by $\rho_{x}^{k}$, and integrating over $(0,1)$ on $x$, we deduce

$$
\begin{aligned}
\frac{\mathrm{d}}{\mathrm{d} t} \int_{0}^{1}\left|\rho_{x}^{k}\right|^{2}(t) \mathrm{d} x & =\int_{0}^{1}\left(\frac{1}{2} u_{x}^{k-1}\left(\rho_{x}^{k}\right)^{2}+\rho^{k} \rho_{x}^{k} u_{x x}^{k-1}\right)(t) \mathrm{d} x \\
& \leqslant C \int_{0}^{1}\left(\left|u_{x}^{k-1}\right|\left|\rho_{x}^{k}\right|^{2}+\rho^{k}\left|\rho_{x}^{k}\right|\left|u_{x x}^{k-1}\right|\right)(t) \mathrm{d} x \\
& \leqslant C\left(\left|u_{x}^{k-1}(t)\right|_{L^{2}(I)}\left|\rho_{x}^{k}(t)\right|_{L^{2}(I)}^{2}+\left|\rho^{k}(t)\right|_{L^{\infty}(I)}\left|\rho_{x}^{k}(t)\right|_{L^{2}(I)}\left|u_{x x}^{k-1}(t)\right|_{L^{2}(I)}\right) .
\end{aligned}
$$

Using Sobolev inequality, we obtain

$$
\frac{\mathrm{d}}{\mathrm{d} t}\left|\rho_{x}^{k}(t)\right|_{L^{2}(I)} \leqslant C\left|\rho^{k}(t)\right|_{H^{1}(I)}\left|u_{x x}^{k-1}(t)\right|_{L^{2}(I)} .
$$

Using (2.28) and (2.29), we have

$$
\begin{aligned}
\frac{\mathrm{d}}{\mathrm{d} t}\left(\left|\rho^{k}(t)\right|_{L^{2}(I)}+\left|\rho_{x}^{k}(t)\right|_{L^{2}(I)}\right) & =\frac{\mathrm{d}}{\mathrm{d} t}\left|\rho^{k}(t)\right|_{H^{1}(I)} \\
& \leqslant C\left|\rho^{k}(t)\right|_{H^{1}(I)}\left|u_{x x}^{k-1}(t)\right|_{L^{2}(I)} .
\end{aligned}
$$


By Gronwall's inequality, we obtain

$$
\sup _{0 \leqslant t \leqslant T}\left|\rho^{k}(t)\right|_{H^{1}(I)} \leqslant\left|\rho_{0}^{k}\right|_{H^{1}(I)} \exp \left(C \int_{0}^{t}\left|u_{x x}^{k-1}(\cdot, s)\right|_{L^{2}(I)} \mathrm{d} s\right) .
$$

Substituting (2.22) into (2.30), we have

$$
\begin{aligned}
\left|\rho^{k}(t)\right|_{H^{1}(I)} & \leqslant C \exp \left(C \int_{0}^{t}\left|u_{x x}^{k-1}(s)\right|_{L^{2}(I)} \mathrm{d} s\right) \\
& \leqslant C \exp \left(C \int_{0}^{t} \Phi_{K}^{\gamma+2}(s) \mathrm{d} s\right) .
\end{aligned}
$$

By (2.10), we have

$$
\left|\rho_{t}^{k}(t)\right|_{L^{2}(I)} \leqslant\left|\rho_{x}^{k}(t)\right|_{L^{2}(I)}\left|u^{k-1}(t)\right|_{L^{\infty}(I)}+\left|\rho^{k}(t)\right|_{L^{\infty}(I)}\left|u_{x}^{k-1}(t)\right|_{L^{2}(I)} \leqslant C \Phi_{K}^{2}(t) .
$$

Combining (2.21), (2.27), (2.31) with (2.22), we obtain

$$
\begin{aligned}
& \left|u_{x}^{k}(t)\right|_{L^{p}(I)}+\left|u_{x x}(t)\right|_{L^{2}(I)}+\left|\rho^{k}(t)\right|_{H^{1}(I)}+\left|\sqrt{\rho^{k}} u_{t}^{k}(t)\right|_{L^{2}(I)}+\int_{0}^{t}\left|u_{x t}^{k}(s)\right|_{L^{2}(I)}^{2} \mathrm{~d} s \\
\leqslant & C_{1} \exp \left(C_{2} \int_{0}^{t} \Phi_{K}^{2 \gamma+7}(s) \mathrm{d} s\right),
\end{aligned}
$$

where $C_{1}, C_{2}$ is a positive constant, depending only on $M^{0}$.

If

$$
\int_{0}^{T} \Phi_{K}^{2 \gamma+7}(s) \mathrm{d} s<1
$$

then we may denote $T=T_{1}$. By inserting the above inequality into (2.32), we can directly obtain (2.34).

On the other hand, if

$$
\int_{0}^{T} \Phi_{K}^{2 \gamma+7}(s) \mathrm{d} s \geqslant 1
$$

then we can find $t_{0} \in(0, T)$ such that

$$
\int_{0}^{t_{0}} \Phi_{K}^{2 \gamma+7}(s) \mathrm{d} s=1 .
$$

Substituting the above identity into (2.32) yields

$$
\sup _{0 \leqslant t \leqslant t_{0}} \Phi_{K}(t) \leqslant C_{1} e^{C_{2}}
$$

and

$$
1=\int_{0}^{t_{0}} \Phi_{K}^{2 \gamma+7}(s) \mathrm{d} s \leqslant \int_{0}^{t_{0}} C_{1}^{2 \gamma+7} e^{(2 \gamma+7) C_{2}} \mathrm{~d} s \leqslant C_{1}^{2 \gamma+7} e^{(2 \gamma+7) C_{2}} t_{0},
$$

then

$$
1 \leqslant C_{1}^{2 \gamma+7} e^{(2 \gamma+7) C_{2}} t_{0}
$$

Here we may denote

$$
T_{1}=C_{1}^{-2 \gamma-7} e^{-(2 \gamma+7) C_{2}},
$$


and thus we deduce

$$
\sup _{0 \leqslant t \leqslant T_{1}} \Phi_{K}(t) \leqslant C_{1} e^{C_{2}} \leqslant C,
$$

where $C$ is a positive constant, depending only on $M_{0}$.

Therefore, for all $1 \leqslant k \leqslant K$, we deduce that

$$
\begin{aligned}
\operatorname{ess} \sup _{0 \leqslant t \leqslant T_{1}}\left(\left|\rho^{k}(t)\right|_{H^{1}(I)}+\left|u^{k}(t)\right|_{W_{0}^{1, p} \cap H^{2}(I)}+\left|\sqrt{\rho^{k}} u_{t}^{k}(t)\right|_{L^{2}(I)}+\left|\rho_{t}^{k}(t)\right|_{L^{2}(I)}\right) \\
\quad+\int_{0}^{T_{1}}\left|u_{x t}^{k}(s)\right|_{L^{2}(I)}^{2} \mathrm{~d} s \leqslant C,
\end{aligned}
$$

where $C$ is a positive constant, depending only on $M^{0}$.

2.2. Convergence of approximate solutions. In this subsection, we will prove that the approximate solutions $\left(\rho^{k}, u^{k}\right)$ strongly converge on the solution of the problem (1.1)(1.2) with positive densities. For this, we denote:

$$
\bar{\rho}^{k+1}=\rho^{k+1}-\rho^{k}, \quad \bar{u}^{k+1}=u^{k+1}-u^{k} .
$$

By (2.11), we get

$$
\begin{aligned}
& \rho^{k+1} \bar{u}_{t}^{k+1}+\rho^{k+1} u^{k} \bar{u}_{x}^{k+1}+\left[\mathrm{E}_{p} u^{k+1}-\mathrm{E}_{p} u^{k}\right]+\left(\pi_{x}^{k+1}-\pi_{x}^{k}\right) \\
= & \bar{\rho}^{k+1}\left(f-u_{t}^{k}-u^{k} u_{x}^{k}\right)-\rho^{k} \bar{u}^{k} u_{x}^{k} .
\end{aligned}
$$

Multiplying (2.36) by $\bar{u}^{k+1}$, integrating over $(0,1)$ on $x$, and using (2.10) and Young's inequality, we have

$$
\begin{aligned}
& \frac{\mathrm{d}}{\mathrm{d} t} \int_{0}^{1} \rho^{k+1}\left|\bar{u}^{k+1}\right|^{2} \mathrm{~d} x+\int_{0}^{1}\left[\mathrm{E}_{p} u^{k+1}-\mathrm{E}_{p} u^{k}\right] \bar{u}^{k+1} \mathrm{~d} x \\
\leqslant & C \int_{0}^{1}\left(\left|\bar{\rho}^{k+1}\right|\left|f-u_{t}^{k}-u^{k} u_{x}^{k}\right|\left|\bar{u}^{k+1}\right|+\rho^{k}\left|\bar{u}^{k}\right|\left|u_{x}^{k}\right|\left|\bar{u}^{k+1}\right|+\left|\pi_{x}^{k+1}-\pi_{x}^{k}\right|^{2}\right) \mathrm{d} x .
\end{aligned}
$$

Let

$$
\Psi(s)=\left(s^{2}+\mu_{0}\right)^{\frac{p-2}{2}} s .
$$

Then

$$
\int_{0}^{1}\left[\mathrm{E}_{p} u^{k+1}-\mathrm{E}_{p} u^{k}\right] \bar{u}^{k+1} \mathrm{~d} x=\int_{0}^{1}\left[\int_{0}^{1} \Psi^{\prime}\left(\theta u^{k+1}+(1-\theta) u^{k}\right) \mathrm{d} \theta\right]\left(\bar{u}_{x}^{k+1}\right)^{2} \mathrm{~d} x .
$$

Since

$$
\begin{aligned}
\Psi^{\prime}(s) & =\left[\left(s^{2}+\mu_{0}\right)^{\frac{p-2}{2}} s\right]^{\prime} \\
& =(p-2)\left(s^{2}+\mu_{0}\right)^{\frac{p-4}{2}} s^{2}+\left(s^{2}+\mu_{0}\right)^{\frac{p-2}{2}} \\
& =\left(s^{2}+\mu_{0}\right)^{\frac{p-4}{2}}\left((p-1) s^{2}+\mu_{0}\right) \\
& \geqslant\left(s^{2}+\mu_{0}\right)^{\frac{p-2}{2}} \\
& \geqslant\left(\mu_{0}\right)^{\frac{p-2}{2}}
\end{aligned}
$$


(2.38) can be written as

$$
\int_{0}^{1}\left[\mathrm{E}_{p} u^{k+1}-\mathrm{E}_{p} u^{k}\right] \bar{u}^{k+1} \mathrm{~d} x \geqslant\left(\mu_{0}\right)^{\frac{p-2}{2}} \int_{0}^{1}\left(\bar{u}_{x}^{k+1}\right)^{2} \mathrm{~d} x .
$$

Substituting it into (2.37), we have

$$
\begin{aligned}
& \frac{\mathrm{d}}{\mathrm{d} t} \int_{0}^{1} \rho^{k+1}\left|\bar{u}^{k+1}\right|^{2} \mathrm{~d} x+\left(\mu_{0}\right)^{\frac{p-2}{2}} \int_{0}^{1}\left(\bar{u}_{x}^{k+1}\right)^{2} \mathrm{~d} x \\
\leqslant & C \int_{0}^{1}\left(\left|\bar{\rho}^{k+1}\right|\left|f-u_{t}^{k}-u^{k} u_{x}^{k}\right|\left|\bar{u}^{k+1}\right|+\rho^{k}\left|\bar{u}^{k}\right|\left|u_{x}^{k}\right|\left|\bar{u}^{k+1}\right|+\left|\pi^{k+1}-\pi^{k}\right|\left|\bar{u}_{x}^{k+1}\right|\right) \mathrm{d} x \\
\leqslant & C\left(\left|\bar{\rho}^{k+1}\right|_{L^{2}(I)}\left|u_{x t}^{k}\right|_{L^{2}(I)}\left|\bar{u}_{x}^{k+1}\right|_{L^{2}(I)}+\left|\bar{\rho}^{k+1}\right|_{L^{2}(I)}\left|u_{x}^{k}\right|_{L^{p}(I)}\left|u_{x x}^{k}\right|_{L^{2}(I)}\left|\bar{u}_{x}^{k+1}\right|_{L^{2}(I)}\right. \\
& +\left|\bar{\rho}^{k+1}\right|_{L^{2}(I)}|f|_{L^{2}(I)}\left|\bar{u}_{x}^{k+1}\right|_{L^{2}(I)}+\left|\rho^{k}\right|_{L^{2}(I)}^{\frac{1}{2}}\left|\sqrt{\rho^{k}} \bar{u}^{k}\right|_{L^{2}(I)}\left|u_{x x}^{k}\right|_{L^{2}(I)}\left|\bar{u}_{x}^{k+1}\right|_{L^{2}(I)} \\
& \left.+\left|\pi^{k+1}-\pi^{k}\right|_{L^{2}(I)}\left|\bar{u}_{x}^{k+1}\right|_{L^{2}(I)}\right),
\end{aligned}
$$

and hence,

$$
\frac{\mathrm{d}}{\mathrm{d} t} \int_{0}^{1} \rho^{k+1}\left|\bar{u}^{k+1}\right|^{2} \mathrm{~d} x+\int_{0}^{1}\left(\bar{u}_{x}^{k+1}\right)^{2} \mathrm{~d} x \leqslant B^{k}(t)\left|\bar{\rho}^{k+1}\right|_{L^{2}(I)}+C\left|\sqrt{\rho^{k}} \bar{u}^{k}\right|_{L^{2}(I)}^{2},
$$

where $B^{k}(t)=C\left(1+|f(t)|_{L^{2}(I)}^{2}+\left|u_{x t}^{k}(t)\right|_{L^{2}(I)}^{2}\right)$. By (2.35), for all $k \geqslant 1$, we have

$$
\int_{0}^{t} B^{k}(t) \mathrm{d} t \leqslant C+C t
$$

where $t<T_{1}$ and $C$ is a positive constant, depending only on $M^{0}$.

By (2.10), we have

$$
\bar{\rho}_{t}^{k+1}+\left(\bar{\rho}^{k+1} u^{k}\right)_{x}+\left(\rho^{k} \bar{u}^{k}\right)_{x}=0 .
$$

Multiplying it by $\bar{\rho}^{k+1}$ and integrating over $(0,1)$, we get

$$
\begin{aligned}
\frac{\mathrm{d}}{\mathrm{d} t}\left|\bar{\rho}^{k+1}\right|_{L^{2}(I)}^{2} & \leqslant C\left|\bar{\rho}^{k+1}\right|_{L^{2}(I)}^{2}\left|u_{x}^{k}\right|_{L^{\infty}(I)}+\left|\rho^{k}\right|_{H^{1}(I)}\left|\bar{u}_{x}^{k}\right|_{L^{2}(I)}\left|\bar{\rho}^{k+1}\right|_{L^{2}(I)} \\
& \leqslant C\left|\bar{\rho}^{k+1}\right|_{L^{2}(I)}^{2}\left|u_{x x}^{k}\right|_{L^{2}(I)}+C_{\eta}\left|\bar{\rho}^{k+1}\right|_{L^{2}(I)}\left|\rho^{k}\right|_{H^{1}(I)}^{2}+\eta\left|\bar{u}_{x}^{k}\right|_{L^{2}(I)}^{2} \\
& \leqslant D_{\eta}^{k}(t)\left|\bar{\rho}^{k+1}\right|_{L^{2}(I)}^{2}+\eta\left|\bar{u}_{x}^{k}\right|_{L^{2}(I)}^{2},
\end{aligned}
$$

where $D_{\eta}^{k}(t)=C\left|u_{x x}^{k}(t)\right|_{L^{2}(I)}+C_{\eta}\left|\rho^{k}(t)\right|_{H^{1}(I)}^{2}$, for all $t \leqslant T_{1}$ and $k \geqslant 1$. By (2.35), we have

$$
\int_{0}^{t} D_{\eta}^{k}(s) \mathrm{d} s \leqslant C+C_{\eta} t
$$

Combining (2.39) with (2.40), we obtain

$$
\begin{aligned}
& \frac{\mathrm{d}}{\mathrm{d} t}\left(\left|\sqrt{\rho^{k+1}} \bar{u}^{k+1}(t)\right|_{L^{2}(I)}^{2}+\left|\bar{\rho}^{k+1}(t)\right|_{L^{2}(I)}^{2}\right)+\left|\bar{u}_{x}^{k+1}(t)\right|_{L^{2}(I)}^{2} \\
\leqslant & C\left|\sqrt{\rho^{k}} \bar{u}^{k}\right|_{L^{2}(I)}^{2}+E_{\eta}(t)\left|\bar{\rho}^{k+1}\right|_{L^{2}(I)}^{2}+\eta\left|\bar{u}^{k}\right|_{L^{2}(I)}^{2},
\end{aligned}
$$


where $E_{\eta}(t)$ depends only on $B^{k}(t)$ and $D_{\eta}(t)$. By (2.35), for all $t \leqslant T_{1}$ and $k \geqslant 1$, we have

$$
\int_{0}^{t} E_{\eta}^{k}(s) \mathrm{d} s \leqslant C+C_{\eta} t
$$

Integrating (2.41) over $(0, t) \subset\left(0, T_{1}\right)$ on the time variable and using Gronwall's inequality, we have

$$
\begin{aligned}
& \left(\left|\sqrt{\rho^{k+1}} \bar{u}^{k+1}(t)\right|_{L^{2}(I)}^{2}+\left|\bar{\rho}^{k+1}(t)\right|_{L^{2}(I)}^{2}\right)+\int_{0}^{t}\left|\bar{u}_{x}^{k+1}(s)\right|_{L^{2}(I)}^{2} \mathrm{~d} s \\
\leqslant & C \exp \left(C_{\eta} t\right) \int_{0}^{t}\left(\left|\sqrt{\rho^{k}} \bar{u}^{k}(s)\right|_{L^{2}(I)}^{2}+\left|\bar{u}_{x}^{k}(s)\right|_{L^{2}(I)}^{2}\right) \mathrm{d} s,
\end{aligned}
$$

where $C$ is a positive constant, depending only on $M^{0}$.

Hence, we choose $\eta>0$, then take enough small $T_{*}$ such that $4 C\left(T_{*}+\eta\right)<1, T_{*}<T_{1}$, and $\exp \left(C_{\eta} T_{*}\right)<2$; then

$$
\begin{gathered}
\left(\left|\sqrt{\rho^{1}} \bar{u}^{1}(t)\right|_{L^{2}(I)}^{2}+\left|\bar{\rho}^{1}(t)\right|_{L^{2}(I)}^{2}\right)+\int_{0}^{t}\left|\bar{u}_{x}^{1}(s)\right|_{L^{2}(I)}^{2} \mathrm{~d} s \\
\leqslant \frac{1}{2} \int_{0}^{t}\left(\left|\sqrt{\rho^{0}} \bar{u}^{0}(s)\right|_{L^{2}(I)}^{2}+\eta\left|\bar{u}_{x}^{0}(s)\right|_{L^{2}(I)}^{2}\right) \mathrm{d} s \\
\left(\left|\sqrt{\rho^{2}} \bar{u}^{2}(t)\right|_{L^{2}(I)}^{2}+\left|\bar{\rho}^{2}(t)\right|_{L^{2}(I)}^{2}\right)+\int_{0}^{t}\left|\bar{u}_{x}^{2}(s)\right|_{L^{2}(I)}^{2} \mathrm{~d} s \\
\leqslant \frac{1}{2} \int_{0}^{t}\left(\left|\sqrt{\rho^{1}} \bar{u}^{1}(s)\right|_{L^{2}(I)}^{2}+\eta\left|\bar{u}_{x}^{1}(s)\right|_{L^{2}(I)}^{2}\right) \mathrm{d} s \\
\vdots \\
\left(\left|\sqrt{\rho^{k+1}} \bar{u}^{k+1}(t)\right|_{L^{2}(I)}^{2}+\left|\bar{\rho}^{k+1}(t)\right|_{L^{2}(I)}^{2}\right)+\int_{0}^{t}\left|\bar{u}_{x}^{k+1}(s)\right|_{L^{2}(I)}^{2} \mathrm{~d} s \\
\leqslant \frac{1}{2} \int_{0}^{t}\left(\left|\sqrt{\rho^{k}} \bar{u}^{k}(s)\right|_{L^{2}(I)}^{2}+\eta\left|\bar{u}_{x}^{k}(s)\right|_{L^{2}(I)}^{2}\right) \mathrm{d} s .
\end{gathered}
$$

Therefore, we combine the above inequalities, and using Gronwall's inequality, we deduce that

$$
\sum_{k=1}^{K}\left[\sup _{0 \leqslant t \leqslant T_{*}}\left(\left|\bar{\rho}^{k+1}(t)\right|_{L^{2}(I)}^{2}+\left|\sqrt{\rho^{k+1}} \bar{u}^{k+1}(t)\right|_{L^{2}(I)}^{2}\right)+\int_{0}^{T_{*}}\left|\bar{u}_{x}^{k+1}(s)\right|_{L^{2}(I)}^{2} \mathrm{~d} s\right]<C,
$$

where $C$ is a positive constant, depending only on $M^{0}$.

For the smooth function $u^{k-1}$, we consider the following initial problem

$$
\left\{\begin{array}{l}
\rho_{t}^{k}+u^{k-1} \rho_{x}^{k}+u_{x}^{k-1} \rho^{k}=0 \\
\left.\rho^{k}\right|_{t=0}=\rho_{0}^{\delta}
\end{array}\right.
$$


Obviously, there is a unique solution $\rho^{k}$ on this above initial problem. Using the method of characteristics, we obtain

$$
\begin{aligned}
& \frac{\mathrm{d} \rho^{k}}{\mathrm{~d} t}=-\rho^{k} u_{x}^{k-1}, \\
& \frac{\mathrm{d} x}{\mathrm{~d} t}=u^{k-1}(x, t), \\
& \left.x\right|_{t=0}=x_{0},\left.\quad \rho^{k}\right|_{t=0}=\rho_{0}^{\delta} .
\end{aligned}
$$

By (2.44) and (2.45), we have

$$
x(t)=x_{0}+\int_{0}^{t} u^{k-1}(x(s), s) \mathrm{d} s=U\left(x_{0}, t\right) .
$$

Using (2.43), we have

$$
\mathrm{d}\left(\ln \rho^{k}\right)=-u_{x}^{k-1} \mathrm{~d} t
$$

then

$$
\rho^{k}(x, t)=\rho_{0}\left(U\left(x_{0}, t\right)\right) \exp \left(-\int_{0}^{t} u_{x}^{k-1}\left(U\left(x_{0}, s\right), s\right) \mathrm{d} s\right) .
$$

Using (2.46) and Sobolev inequality, we have

$$
\rho^{k}(x, t) \geqslant \delta \exp \left[-\int_{0}^{T}\left|u_{x}^{k-1}(\cdot, s)\right|_{L^{\infty}(I)} \mathrm{d} s\right] .
$$

Then for all $t \in\left(0, T_{*}\right)$, we get

$$
\rho^{k+1} \geqslant \delta C^{-1}>0 .
$$

Hence, by (2.42), we get the following convergence: as $k \rightarrow+\infty$,

$$
\begin{aligned}
& u^{k} \rightarrow u \quad L^{\infty}\left(0, T_{*} ; L^{2}(I)\right) \cap L^{2}\left(0, T_{*} ; H_{0}^{1}(I)\right), \\
& \rho^{k} \rightarrow \rho \quad L^{\infty}\left(0, T_{*} ; L^{2}(I)\right) .
\end{aligned}
$$

By virtue of the lower semi-continuity of various norms, we deduce that $(\rho, u)$ satisfies the following uniform estimate:

$$
\begin{aligned}
\operatorname{ess} \sup _{0 \leqslant t \leqslant T_{*}}\left(|\rho(t)|_{H^{1}(I)}+|u(t)|_{W_{0}^{1, p}(I) \cap H^{2}(I)}+\left|\sqrt{\rho} u_{t}(t)\right|_{L^{2}(I)}+\left|\rho_{t}(t)\right|_{L^{2}(I)}\right) \\
+\int_{0}^{T_{*}}\left|u_{x t}(s)\right|_{L^{2}(I)}^{2} \mathrm{~d} s \leqslant C
\end{aligned}
$$

where $C$ is a positive constant, depending only on $M_{0}$.

2.3. The proof of Proposition 2.1. In this subsection, we will prove Proposition 2.1 in order to use (2.48) and (2.49); namely, under the assumption of Proposition 2.1 we will prove that $(\rho, u)$ is a unique solution of the initial boundary value problem (1.1)-1.2) with positive densities. For this, we only need to prove that $(\rho, u)$ satisfies the following properties: 
(i)

$$
\begin{aligned}
& \rho \in C\left(\left[0, T_{*}\right] ; H^{1}(I)\right), \quad \rho_{t} \in C\left(\left[0, T_{*}\right] ; L^{2}(I)\right), \quad \sqrt{\rho} u_{t} \in L^{\infty}\left(0, T_{*} ; L^{2}(I)\right), \\
& u \in C\left(\left[0, T_{*}\right] ; H_{0}^{1}(I)\right) \cap L^{\infty}\left(0, T_{*} ; H^{2}(I)\right), \quad u_{t} \in L^{2}\left(0, T_{*} ; H_{0}^{1}(I)\right), \\
& \left(\left(u_{x}^{2}+\mu_{0}\right)^{\frac{p-2}{2}} u_{x}\right)_{x} \in C\left(\left[0, T_{*}\right] ; L^{2}(I)\right) .
\end{aligned}
$$

(ii) For all $\varphi \in C\left(\left[0, T_{*}\right] ; H^{1}(I)\right), \varphi_{t} \in L^{\infty}\left(0, T_{*} ; L^{2}(I)\right)$, for a.e. $t \in\left(0, T_{*}\right)$, the following identity holds:

$$
\int_{0}^{1} \rho(x, t) \varphi(x, t) \mathrm{d} x-\int_{0}^{t} \int_{0}^{1}\left(\rho \varphi_{t}+\rho u \varphi_{x}\right) \mathrm{d} x \mathrm{~d} s=\int_{0}^{1} \rho_{0} \varphi(x, 0) \mathrm{d} x .
$$

(iii) For all $\phi \in C\left(\left[0, T_{*}\right] ; H_{0}^{1}(I)\right) \cap L^{\infty}\left(0, T_{*} ; H^{2}(I)\right), \phi_{t} \in L^{2}\left(0, T_{*} ; H_{0}^{1}(I)\right)$, for a.e. $t \in\left(0, T_{*}\right)$, the following identity holds:

$$
\begin{aligned}
& \int_{0}^{1} \rho u \phi(x, t) \mathrm{d} x-\int_{0}^{t} \int_{0}^{1}\left\{\rho u \phi_{t}+\rho(u)^{2} \phi_{x}+\pi \phi_{x}-\left(u_{x}^{2}+\mu_{0}\right)^{\frac{p-2}{2}} u_{x} \phi_{x}\right\}(x, s) \mathrm{d} x \mathrm{~d} s \\
& =\int_{0}^{1} \rho_{0} u_{0} \phi(x, 0) \mathrm{d} x+\int_{0}^{t} \int_{0}^{1} \rho f \phi(x, s) \mathrm{d} x \mathrm{~d} s .
\end{aligned}
$$

Since $\left(\rho^{k}, u^{k}\right)$ is a smooth solution of (2.10) 2.12), it naturally satisfies the following identities:

$$
\int_{0}^{1} \rho^{k}(x, t) \varphi(x, t) \mathrm{d} x-\int_{0}^{t} \int_{0}^{1}\left(\rho^{k} \varphi_{t}+\rho^{k} u^{k-1} \varphi_{x}\right) \mathrm{d} x \mathrm{~d} s=\int_{0}^{1} \rho_{0} \varphi(x, 0) \mathrm{d} x
$$

where $\varphi \in C\left(\left[0, T_{*}\right] ; H^{1}(I)\right), \varphi_{t} \in L^{\infty}\left(0, T_{*} ; L^{2}(I)\right)$, and

$$
\begin{aligned}
& \int_{0}^{1} \rho^{k} u \phi(x, t) \mathrm{d} x-\int_{0}^{t} \int_{0}^{1}\left\{\rho^{k} u^{k} \phi_{t}+\rho^{k}\left(u^{k}\right)^{2} \phi_{x}+\pi^{k} \phi_{x}-\left(u_{x}^{2}+\mu_{0}\right)^{\frac{p-2}{2}} u_{x} \phi_{x}\right\}(x, s) \mathrm{d} x \mathrm{~d} s \\
& =\int_{0}^{1} \rho_{0} u_{0} \phi(x, 0) \mathrm{d} x+\int_{0}^{t} \int_{0}^{1} \rho^{k} f \phi(x, s) \mathrm{d} x \mathrm{~d} s,
\end{aligned}
$$

where $\phi \in C\left(\left[0, T_{*}\right] ; H_{0}^{1}(I)\right) \cap L^{\infty}\left(0, T_{*} ; H^{2}(I)\right), \phi_{t} \in L^{2}\left(0, T_{*} ; H_{0}^{1}(I)\right)$. Hence, if we want to prove (2.50), (2.51), we only need to prove that, as $k \rightarrow \infty$,

$$
\begin{aligned}
\sum_{i=1}^{3} \mathrm{I}_{i}^{k}= & \int_{0}^{1}\left[\rho^{k}(x, t)-\rho(x, t)\right] \varphi(x, t) \mathrm{d} x \\
& -\int_{0}^{t} \int_{0}^{1}\left[\left(\rho^{k}-\rho\right) \varphi_{t}+\left(\rho^{k} u^{k-1}-\rho u\right) \varphi_{x}\right] \mathrm{d} x \mathrm{~d} s \rightarrow 0
\end{aligned}
$$


and

$$
\begin{aligned}
\sum_{i=1}^{6} \mathrm{II}_{i}^{k}= & \int_{0}^{1}\left(\rho^{k}(x, t) u^{k}(x, t)-\rho(x, t) u(x, t)\right) \phi(x, t) \mathrm{d} x \\
& -\int_{0}^{t} \int_{0}^{1}\left\{\left(\rho^{k} u^{k}-\rho u\right) \phi_{t}+\left(\rho^{k}\left(u^{k}\right)^{2}-\rho(u)^{2}\right) \phi_{x}+\left(\pi^{k}-\pi\right) \phi_{x}\right. \\
& \left.-\left(\left(u^{k}\right)_{x}^{2}+\mu_{0}\right)^{\frac{p-2}{2}} u_{x}^{k}-\left(u_{x}^{2}+\mu_{0}\right)^{\frac{p-2}{2}} u_{x} \phi_{x}\right\} \mathrm{d} x \mathrm{~d} s \\
& -\int_{0}^{t} \int_{0}^{1}\left(\rho^{k}-\rho\right) f \phi(x, s) \mathrm{d} x \mathrm{~d} s \rightarrow 0 .
\end{aligned}
$$

We first calculate the fifth term of (2.53):

$$
\mathrm{II}_{5}^{\mathrm{k}}=\int_{0}^{t} \int_{0}^{1}\left[\left(\left(u^{k}\right)_{x}^{2}+\mu_{0}\right)^{\frac{p-2}{2}} u_{x}^{k}-\left(u_{x}^{2}+\mu_{0}\right)^{\frac{p-2}{2}} u_{x}\right] \phi_{x} \mathrm{~d} x \mathrm{~d} s,
$$

where $\phi \in C\left(\left[0, T_{*}\right] ; H_{0}^{1}(I)\right) \cap L^{\infty}\left(0, T_{*} ; H^{2}(I)\right), \phi_{t} \in L^{2}\left(0, T_{*} ; H_{0}^{1}(I)\right)$.

Let

$$
\omega(s)=\left(s^{2}+\mu_{0}\right)^{\frac{p-2}{2}} s .
$$

Then we obtain

$$
\begin{aligned}
\mathrm{II}_{5}^{\mathrm{k}} \leqslant & \int_{0}^{t} \int_{0}^{1}\left|\omega\left(u_{x}^{k}\right)-\omega\left(u_{x}\right)\right|\left|\phi_{x}\right| \mathrm{d} x \mathrm{~d} s \\
= & \int_{0}^{t} \int_{0}^{1}\left|\int_{0}^{1} \omega^{\prime}\left(\theta u_{x}^{k}+(1-\theta) u_{x}\right) \mathrm{d} \theta\left(u_{x}^{k}-u_{x}\right)\right|\left|\phi_{x}\right| \mathrm{d} x \mathrm{~d} s \\
\leqslant & \int_{0}^{t} \int_{0}^{1}\left|\int_{0}^{1} \omega^{\prime}\left(\theta u_{x}^{k}+(1-\theta) u_{x}\right) \mathrm{d} \theta\right|\left|u_{x}^{k}-u_{x}\right|\left|\phi_{x}\right| \mathrm{d} x \mathrm{~d} s \\
\leqslant & \int_{0}^{t} \int_{0}^{1}\left|\int_{0}^{1}\left(\mu_{0}+\left(\theta u_{x}^{k}+(1-\theta) u_{x}\right)^{2}\right)^{\frac{p-2}{2}} \mathrm{~d} \theta\right|\left|u_{x}^{k}-u_{x}\right|\left|\phi_{x}\right| \mathrm{d} x \mathrm{~d} s \\
\leqslant & \left|\phi_{x}\right|_{L^{\infty}\left(0, T_{*} ; L^{\infty}(I)\right)}\left(\mu_{0}+2\left|u_{x}^{k}\right|_{L^{\infty}\left(0, T_{*} ; L^{\infty}(I)\right)}^{2}+2\left|u_{x}\right|_{L^{\infty}\left(0, T_{*} ; L^{\infty}(I)\right)}^{2}\right)^{\frac{p-2}{2}} \\
& \cdot \int_{0}^{t}\left(\int_{0}^{1}\left|u_{x}^{k}-u_{x}\right|^{2} \mathrm{~d} x\right)^{\frac{1}{2}} \mathrm{~d} s \\
\leqslant & C \int_{0}^{t}\left(\int_{0}^{1}\left|u_{x}^{k}-u_{x}\right|^{2} \mathrm{~d} x\right)^{\frac{1}{2}} \mathrm{~d} s,
\end{aligned}
$$

where $C$ is a positive constant, depending only on $M_{*}$. Here and below, we denote

$$
\begin{aligned}
M_{*}= & 1+M^{0}+\left|\phi_{x}\right|_{L^{\infty}\left(0, T_{*} ; L^{\infty}(I)\right)}+\left|\phi_{t}\right|_{L^{\infty}\left(0, T_{*} ; L^{2}(I)\right)} \\
& +\left|\varphi_{x}\right|_{L^{\infty}\left(0, T_{*} ; L^{2}(I)\right)}+\left|\varphi_{t}\right|_{L^{\infty}\left(0, T_{*} ; L^{2}(I)\right)}+|f|_{L^{\infty}\left(0, T_{*} ; L^{2}(I)\right)} .
\end{aligned}
$$


We then calculate the second and third terms of (2.53):

$$
\mathrm{II}_{2}^{\mathrm{k}}=\int_{0}^{t} \int_{0}^{1}\left(\rho^{k} u^{k}-\rho u\right) \phi_{t} \mathrm{~d} x \mathrm{~d} s
$$

and

$$
\mathrm{II}_{3}^{\mathrm{k}}=\int_{0}^{t} \int_{0}^{1}\left(\rho^{k}\left(u^{k}\right)^{2}-\rho(u)^{2}\right) \phi_{x} \mathrm{~d} x \mathrm{~d} s .
$$

For (2.56), we obtain

$$
\begin{aligned}
\mathrm{II}_{2}^{\mathrm{k}} \leqslant & \int_{0}^{t} \int_{0}^{1}\left|\rho^{k} u^{k}-\rho u\right|\left|\phi_{t}\right| \mathrm{d} x \mathrm{~d} s \\
\leqslant & \int_{0}^{t} \int_{0}^{1}\left|\rho^{k}-\rho\right||u|\left|\phi_{t}\right| \mathrm{d} x \mathrm{~d} s+\int_{0}^{t} \int_{0}^{1}\left|\rho^{k}\right|\left|u^{k}-u\right|\left|\phi_{t}\right| \mathrm{d} x \mathrm{~d} s \\
\leqslant & |u|_{L^{\infty}\left(0, t ; L^{\infty}(I)\right)}\left|\phi_{t}\right|_{L^{\infty}\left(0, t ; L^{2}(I)\right)} \int_{0}^{t}\left(\int_{0}^{1}\left|\rho^{k}-\rho\right|^{2} \mathrm{~d} x\right)^{1 / 2} \mathrm{~d} s \\
& +\left|\rho^{k}\right|_{L^{\infty}\left(0, t ; L^{\infty}(I)\right)}\left|\phi_{t}\right|_{L^{\infty}\left(0, t ; L^{2}(I)\right)} \int_{0}^{t}\left(\int_{0}^{1}\left|u^{k}-u\right|^{2} \mathrm{~d} x\right)^{1 / 2} \mathrm{~d} s \\
& \leqslant C\left[\int_{0}^{t}\left(\int_{0}^{1}\left|\rho^{k}-\rho\right|^{2} \mathrm{~d} x\right)^{1 / 2} \mathrm{~d} s+\int_{0}^{t}\left(\int_{0}^{1}\left|u^{k}-u\right|^{2} \mathrm{~d} x\right)^{1 / 2} \mathrm{~d} s\right],
\end{aligned}
$$

where $C$ is a positive constant, depending only on $M_{*}$.

Now we calculate (2.57):

$$
\begin{aligned}
\mathrm{II}_{3}^{\mathrm{k}} \leqslant & \int_{0}^{t} \int_{0}^{1}\left|\rho^{k}\left(u^{k}\right)^{2}-\rho(u)^{2}\right|\left|\phi_{x}\right| \mathrm{d} x \mathrm{~d} s \\
\leqslant & \int_{0}^{t} \int_{0}^{1}\left|\rho^{k} u^{k}-\rho u\right|\left|u^{k}\right|\left|\phi_{x}\right| \mathrm{d} x \mathrm{~d} s+\int_{0}^{t} \int_{0}^{1}\left|u^{k}-u\right||\rho u|\left|\phi_{x}\right| \mathrm{d} x \mathrm{~d} s \\
\leqslant & \left|u^{k}\right|_{L^{\infty}\left(0, t ; L^{\infty}(I)\right)}\left|\phi_{x}\right|_{L^{\infty}\left(0, t ; L^{\infty}(I)\right)} \int_{0}^{t} \int_{0}^{1}\left|\rho^{k} u^{k}-\rho u\right| \mathrm{d} x \mathrm{~d} s \\
& +\left|\rho u \phi_{x}\right|_{L^{\infty}\left(0, t ; L^{\infty}(I)\right)} \int_{0}^{t} \int_{0}^{1}\left|u^{k}-u\right| \mathrm{d} x \mathrm{~d} s \\
\leqslant & {\left[\int_{0}^{t}\left(\int_{0}^{1}\left|\rho^{k}-\rho\right|^{2} \mathrm{~d} x\right)^{1 / 2} \mathrm{~d} s+\int_{0}^{t}\left(\int_{0}^{1}\left|u^{k}-u\right|^{2} \mathrm{~d} x\right)^{1 / 2} \mathrm{~d} s\right], }
\end{aligned}
$$

where $C$ is a positive constant, depending only on $M_{*}$. 
Finally, we calculate the remaining terms:

$$
\begin{aligned}
& \mathrm{I}_{1}^{k} \leqslant \int_{0}^{1}\left[\rho^{k}(x, t)-\rho(x, t)\right] \varphi(x, t) \mathrm{d} x \\
& \leqslant|\varphi|_{L^{\infty}(I)} \int_{0}^{1}\left|\rho^{k}(x, t)-\rho(x, t)\right| \mathrm{d} x \\
& \leqslant C\left(\int_{0}^{1}\left|\rho^{k}(x, t)-\rho(x, t)\right|^{2} \mathrm{~d} x\right)^{\frac{1}{2}} \text {. } \\
& \mathrm{I}_{2}^{k}+\mathrm{I}_{3}^{k} \leqslant \int_{0}^{t} \int_{0}^{1}\left(\left|\rho^{k}-\rho\right|\left|\varphi_{t}\right|+\left|\rho^{k} u^{k}-\rho u\right|\left|\varphi_{x}\right|\right) \mathrm{d} x \mathrm{~d} s \\
& \leqslant\left|\varphi_{t}\right|_{L^{\infty}\left(0, t ; L^{2}(I)\right)} \int_{0}^{t}\left(\int_{0}^{1}\left|\rho^{k}-\rho\right|^{2} \mathrm{~d} x\right)^{\frac{1}{2}} \mathrm{~d} s \\
& +\left|\varphi_{x}\right|_{L^{\infty}\left(0, t ; L^{2}(I)\right)} \int_{0}^{t}\left(\int_{0}^{1}\left|\rho^{k} u^{k}-\rho u\right|^{2} \mathrm{~d} x\right)^{\frac{1}{2}} \mathrm{~d} s \\
& \leqslant C\left[\int_{0}^{t}\left(\int_{0}^{1}\left|\rho^{k}-\rho\right|^{2} \mathrm{~d} x\right)^{\frac{1}{2}} \mathrm{~d} s+\int_{0}^{t}\left(\int_{0}^{1}\left|u^{k}-u\right|^{2} \mathrm{~d} x\right)^{\frac{1}{2}} \mathrm{~d} s\right] . \\
& \mathrm{II}_{1}^{k} \leqslant \int_{0}^{1}\left|\rho^{k} u^{k}-\rho u \| \phi(x, t)\right| \mathrm{d} x \\
& \leqslant|\phi(x, t)|_{L^{2}(I)}\left(\int_{0}^{1}\left|\rho^{k} u^{k}-\rho u\right|^{2} \mathrm{~d} x\right)^{\frac{1}{2}} \\
& \leqslant C\left[\left(\int_{0}^{1}\left|\rho^{k}-\rho\right|^{2} \mathrm{~d} x\right)^{\frac{1}{2}}+\left(\int_{0}^{1}\left|u^{k}-u\right|^{2} \mathrm{~d} x\right)^{\frac{1}{2}}\right] . \\
& \mathrm{II}_{4}^{k} \leqslant \int_{0}^{t} \int_{0}^{1}\left|\pi^{k}-\pi\right|\left|\phi_{x}\right| \mathrm{d} x \mathrm{~d} s \\
& \leqslant\left|\phi_{x}\right|_{L^{\infty}\left(0, t ; L^{2}(I)\right)} \int_{0}^{t}\left(\int_{0}^{1}\left|\pi^{k}-\pi\right|^{2} \mathrm{~d} x\right)^{\frac{1}{2}} \mathrm{~d} s \\
& \leqslant C \int_{0}^{t}\left(\int_{0}^{1}\left|\pi^{k}-\pi\right|^{2} \mathrm{~d} x\right)^{\frac{1}{2}} \mathrm{~d} s \\
& \mathrm{II}_{6}^{k} \leqslant \int_{0}^{t} \int_{0}^{1}\left|\rho^{k}-\rho\right||f \phi(x, s)| \mathrm{d} x \mathrm{~d} s \\
& \leqslant|f|_{L^{2}\left(0, t ; L^{2}(I)\right)}|\phi(x, s)|_{L^{\infty}\left(0, t ; L^{2}(I)\right)} \int_{0}^{t}\left(\int_{0}^{1}\left|\rho^{k}-\rho\right|^{2} \mathrm{~d} x\right)^{\frac{1}{2}} \mathrm{~d} s \\
& \leqslant C \int_{0}^{t}\left(\int_{0}^{1}\left|\rho^{k}-\rho\right|^{2} \mathrm{~d} x\right)^{\frac{1}{2}} \mathrm{~d} s
\end{aligned}
$$


Since the initial value is independent of $k$, as $k \rightarrow+\infty,\left(\rho_{0}, u_{0}\right)$ still satisfies the following equation:

$$
\left(\left(u_{0 x}^{2}+\mu_{0}\right)^{\frac{p-2}{2}} u_{0 x}\right)_{x}=\pi_{x}\left(\rho_{0}\right)-\rho_{0}^{\frac{1}{2}} g .
$$

Hence, we deduce that

$$
\begin{aligned}
& \sum_{i=1}^{3}\left|\mathrm{I}_{i}^{k}\right|+\sum_{i=1}^{6}\left|\mathrm{I} \mathrm{I}_{i}^{k}\right| \\
\leqslant & C\left\{\int_{0}^{t}\left(\int_{0}^{1}\left|u_{x}^{k}-u_{x}\right|^{2} \mathrm{~d} x\right)^{\frac{1}{2}} \mathrm{~d} s+\int_{0}^{t}\left(\int_{0}^{1}\left|u_{x}^{k}-u_{x}\right|^{2} \mathrm{~d} x\right)^{\frac{p-1}{2}} \mathrm{~d} s\right. \\
& +\int_{0}^{t}\left(\int_{0}^{1}\left|\rho^{k}-\rho\right|^{2} \mathrm{~d} x\right)^{\frac{1}{2}}+\int_{0}^{t}\left(\int_{0}^{1}\left|\pi^{k}-\pi\right|^{2} \mathrm{~d} x\right)^{\frac{1}{2}} \mathrm{~d} s \\
& \left.+\left(\int_{0}^{1}\left|\rho^{k}-\rho\right|^{2} \mathrm{~d} x\right)^{\frac{1}{2}}+\left(\int_{0}^{1}\left|u^{k}-u\right|^{2} \mathrm{~d} x\right)^{\frac{1}{2}}\right\},
\end{aligned}
$$

where $C$ is a positive constant, depending only on $M_{*}$. By the convergence (2.48), we see, as $k \rightarrow+\infty$, that the right-hand side of the above inequality vanishes; namely, $(\rho, u)$ satisfies the integration equations (2.50) and (2.51).

To finish the proof of existence, we still need to prove that $(\rho, u)$ satisfies the following properties:

$$
\begin{gathered}
\rho \in C\left(\left[0, T_{*}\right] ; H^{1}(I)\right), \quad \rho_{t} \in C\left(\left[0, T_{*}\right] ; L^{2}(I)\right), \\
u \in C\left(\left[0, T_{*}\right] ; H_{0}^{1}(I)\right), \quad \mathrm{E}_{p} u \in C\left(\left[0, T_{*}\right] ; L^{2}(I)\right) .
\end{gathered}
$$

First, we prove

$$
\rho \in C\left(\left[0, T_{*}\right] ; H^{1}(I)\right) .
$$

Since $\rho$ satisfies the following regularity:

$$
\text { ess } \sup _{0 \leqslant t \leqslant T_{*}}\left(\left|\sqrt{\rho} u_{t}(t)\right|_{L^{2}(I)}^{2}+|\rho(t)|_{H^{1}(I)}+\left|\rho_{t}(t)\right|_{L^{2}(I)}\right) \leqslant C,
$$

by the embedding theorem (see [3], Chapter I), we obtain

$$
\rho \in C\left(\left[0, T_{*}\right] ; L^{2}(I)\right) .
$$

Now, we will prove

$$
\rho \in C\left(\left[0, T_{*}\right] ; H^{1}(I)\right) .
$$

Using (2.10), we have

$$
\frac{\mathrm{d}}{\mathrm{d} t}\left|\rho_{x}^{k}(t)\right|_{L^{2}(I)}^{2} \leqslant C\left|\rho_{x}^{k}(0)\right|_{L^{2}(I)}^{2}\left|u_{x}^{k-1}(t)\right|_{H^{1}(I)}
$$

and

$$
\left|\rho_{x}^{k}(t)\right|_{L^{2}(I)}^{2} \leqslant\left|\rho_{x}(0)\right|_{L^{2}(I)}^{2}+C t .
$$

By the lower semi-continuity of various norms, we have

$$
\left|\rho_{x}(t)\right|_{L^{2}(I)}^{2} \leqslant\left|\rho_{x}(0)\right|_{L^{2}(I)}^{2}+C t .
$$


Taking limits on the above inequality, letting $t \rightarrow 0$, we have

$$
\limsup _{t \rightarrow 0}\left|\rho_{x}(t)\right|_{L^{2}(I)}^{2} \leqslant\left|\rho_{x}(0)\right|_{L^{2}(I)}^{2} .
$$

Hence, using the strong convergence for the space $L^{2}$ (see [18]), we have

$$
\lim _{t \rightarrow 0^{+}}\left|\rho_{x}(t)-\rho_{x}(0)\right|_{L^{2}(I)}^{2}=0 .
$$

For each fixed $t_{0} \in[0, t]$, the function $\hat{\rho}=\hat{\rho}(x, t)=\hat{\rho}\left(x, \pm t+t_{0}\right)$ is a unique strong solution to the similar initial problem

$$
\left\{\begin{array}{l}
\hat{\rho}_{t}+(\hat{\rho} \hat{v})_{x}=0, \\
\hat{\rho}(0)=\rho\left(t_{0}\right),
\end{array}\right.
$$

where $\hat{v}=\hat{v}(x, t)= \pm v\left(x, \pm t+t_{0}\right)$. Therefore, $\rho_{x} \in C\left(\left[0, T_{*}\right] ; L^{2}(I)\right)$. Then we have $\rho \in C\left(\left[0, T_{*}\right] ; H^{1}(I)\right)$.

By virtue of (2.49) and the embedding theorem (see [3]), we obtain

$$
u \in C\left([0, T] ; H_{0}^{1}(I)\right) .
$$

Hence, we only need to prove that $u$ satisfies the following properties:

$$
\mathrm{E}_{p} u \in C\left(\left[0, T_{*}\right] ; L^{2}(I)\right) .
$$

By the momentum equation of (2.11), $\forall \omega \in H_{0}^{1}(I)$, we get

$$
\begin{aligned}
\left(\rho u_{t}, \omega\right)_{L^{2}(I)} & =\left(-\rho u u_{x}+\left(\left(\left|u_{x}\right|^{2}+\mu_{0}\right)^{\frac{p-2}{2}} u_{x}\right)_{x}-\pi_{x}+\rho f, \omega\right)_{L^{2}(I)} \\
& =\left(-\rho u u_{x}+\rho f, \omega\right)_{L^{2}(I)}-\left(\left(\left|u_{x}\right|^{2}+\mu_{0}\right)^{\frac{p-2}{2}} u_{x}-\pi, \omega_{x}\right)_{L^{2}(I)} ;
\end{aligned}
$$

then

$$
\frac{\mathrm{d}}{\mathrm{d} t}\left(\rho u_{t}, \omega\right)_{L^{2}(I)}=\left(\left(-\rho u u_{x}+\rho f\right)_{t}, \omega\right)_{L^{2}(I)}-\left(\left(\left(\left|u_{x}\right|^{2}+\mu_{0}\right)^{\frac{p-2}{2}} u_{x}-\pi\right)_{t}, \omega_{x}\right)_{L^{2}(I)} .
$$

By (2.49), we obtain $A(t)|\omega|_{H_{0}^{1}(I)}$ is the upper boundary for the right-hand side of (2.61), where $A(t) \in L^{2}\left(0, T_{*}\right)$. Then $\left(\rho u_{t}\right)_{t} \in L^{2}\left(0, T ; H^{-1}(I)\right)$ (see 20], third chapter lemma 1.1). Hence, $\rho u_{t} \in L^{2}\left(0, T ; H_{0}^{1}(I)\right)$. By the embedding theorem, we obtain $\rho u_{t} \in$ $C\left(\left[0, T_{*}\right] ; L^{2}(I)\right)$.

Therefore, we obtain, for all $t \in[0, T]$, that $u=u(t) \in H_{0}^{1}(I) \cap H^{2}(I)$ is a solution of the following elliptical equation (see [19]):

$$
\mathrm{E}_{p} u=G-\rho u u_{x},
$$

where $G=\rho f-\rho u_{t}-\pi_{x} \in C\left([0, T] ; L^{2}(I)\right)$.

Since

$$
\begin{aligned}
& \left|\mathrm{E}_{p} u(t)-\mathrm{E}_{p} u(s)\right|_{L^{2}(I)} \\
= & \left|G(t)-\rho u u_{x}(t)-\left(G(s)-\rho u u_{x}(s)\right)\right|_{L^{2}(I)} \\
\leqslant & \left|\rho u u_{x}(t)-\rho u u_{x}(s)\right|_{L^{2}(I)}+|G(s)-G(t)|_{L^{2}(I)},
\end{aligned}
$$


the first term of 2.62 may be written as

$$
\begin{aligned}
& \left|\rho u u_{x}(t)-\rho u u_{x}(s)\right|_{L^{2}(I)} \\
\leqslant & \left|(\rho(t)-\rho(s)) u(t) u_{x}(t)\right|_{L^{2}(I)}+\left|\rho(s)(u(t)-u(s)) u_{x}(t)\right|_{L^{2}(I)} \\
& +\left|\rho(s) u(s) \cdot\left(u_{x}(t)-u_{x}(s)\right)\right|_{L^{2}(I)} \\
\leqslant & \left(|\rho(t)-\rho(s)|_{L^{2}(I)}\left|u_{x}(t)\right|_{L^{p}(I)}+|\rho(s)|_{L^{2}(I)}\left|u_{x}(s)-u_{x}(t)\right|_{L^{2}(I)}\right)\left|u_{x}(t)\right|_{L^{\infty}(I)} \\
& +|\rho(s)|_{L^{\infty}(I)}\left|u_{x}(s)\right|_{L^{\infty}(I)}\left|u_{x}(t)-u_{x}(s)\right|_{L^{2}(I)} \\
\leqslant & C\left(|\rho(t)-\rho(s)|_{L^{2}(I)}+|u(t)-u(s)|_{H_{0}^{1}(I)}\right)
\end{aligned}
$$

Hence,

$$
\lim _{t \rightarrow s}\left|\mathrm{E}_{p} u(t)-\mathrm{E}_{p} u(s)\right|_{L^{2}(I)}=0 .
$$

This proves the continuity of $\mathrm{E}_{p} u(t)$ in $L^{2}(I)$.

The proof of uniqueness is the same as Theorem 1.2 , we omit it here. Hence, Proposition 2.1 is proved.

3. The proof of Theorem 1.2. In this section, we will prove the main Theorem 1.2 . We allow the problem to have the state of vacuum in Theorem 1.2 and in the previous section, we assumed that the initial density is positive and we obtained a similar result, so we hope to use the result to prove Theorem 1.2. First, we regularize the known functions of the original problem (1.1)-(1.2) such that they satisfy the conditions of Proposition 2.1. Using Proposition 2.1, we can prove that the limit of solutions of the regulation problem is the solution to the original problem (1.1)-(1.2).

3.1. The proof of existence. Assume that $\rho_{0}$ is sufficiently smooth; then, for each small $\delta>0$, let $\rho_{0}^{\delta}=J_{\delta} * \rho_{0}+\delta$. $J_{\delta}$ is a mollifier on $I$, and $u_{0}^{\delta} \in H_{0}^{1}(I) \cap H^{2}(I)$ is a solution of the following boundary value problem (see [19], Chapter II):

$$
\begin{aligned}
& -\left[\left(\left(u_{0 x}^{\delta}\right)^{2}+\mu_{0}\right)^{\frac{p-2}{2}} u_{0 x}^{\delta}\right]_{x}=-\pi_{x}\left(\rho_{0}^{\delta}\right)+\left(\rho_{0}^{\delta}\right)^{\frac{1}{2}} g_{\delta} \quad x \in(0,1), \\
& u_{0}^{\delta}(0)=u_{0}^{\delta}(1)=0
\end{aligned}
$$

where $g_{\delta} \in C_{0}^{\infty}(I)$ and satisfies

$$
\left|g_{\delta}\right|_{L^{2}(I)} \leqslant|g|_{L^{2}(I)}, \quad \lim _{\delta \rightarrow 0}\left|g_{\delta}-g\right|_{L^{2}(I)}=0 .
$$

With $\rho_{0}^{\delta}=J_{\delta} * \rho_{0}+\delta$, there exists a subsequence $\left\{\rho^{\delta_{j}}\right\}$ of $\left\{\rho^{\delta}\right\}$ that satisfies the following convergence: as $\delta_{j} \rightarrow 0$,

$$
-\pi_{x}\left(\rho_{0}^{\delta_{j}}\right)+\left(\rho_{0}^{\delta_{j}}\right)^{\frac{1}{2}} g_{\delta_{j}} \rightarrow-\pi_{x}\left(\rho_{0}\right)+\left(\rho_{0}\right)^{\frac{1}{2}} g \quad \text { in } \quad L^{2}(I) .
$$

By (3.64), there exists a subsequence $\left\{u_{0}^{\delta_{j}}\right\}$ of $\left\{u_{0}^{\delta}\right\}$ such that as $\delta_{j} \rightarrow 0$,

$$
\left[\left(\left(u_{0 x}^{\delta_{j}}\right)^{2}+\mu_{0}\right)^{\frac{p-2}{2}} u_{0 x}^{\delta_{j}}\right]_{x} \rightarrow\left[\left(\left(u_{0 x}\right)^{2}+\mu_{0}\right)^{\frac{p-2}{2}} u_{0 x}\right]_{x} \quad \text { in } \quad L^{2}(I) .
$$


Hence, $\left(\rho_{0}, u_{0}\right)$ satisfies the following equation:

$$
-\left[\left(\left(u_{0 x}\right)^{2}+\mu_{0}\right)^{\frac{p-2}{2}} u_{0 x}\right]_{x}=-\pi_{x}\left(\rho_{0}\right)+\left(\rho_{0}\right)^{\frac{1}{2}} g \text { for a.e. } x \in \mathrm{I} .
$$

Taking $f^{\delta} \in C^{\infty}\left(\bar{\Omega}_{T}\right), f^{\delta}(0, t)=f^{\delta}(1, t)=0$, satisfying

$$
\begin{aligned}
& \left|f^{\delta}\right|_{L^{\infty}\left(0, T ; L^{2}(I)\right)} \leqslant C|f|_{L^{\infty}\left(0, T ; L^{2}(I)\right)}, \\
& \left|f^{\delta}\right|_{L^{\infty}\left(0, T ; L^{2}(I)\right)} \leqslant C|f|_{L^{\infty}\left(0, T ; L^{2}(I)\right)},
\end{aligned}
$$

and as $\delta \rightarrow 0$, there hold the following limits:

$$
\begin{aligned}
& \left|f^{\delta}-f\right|_{L^{\infty}\left(0, T ; L^{2}(I)\right)} \rightarrow 0, \\
& \left|f_{t}^{\delta}-f_{t}\right|_{L^{\infty}\left(0, T ; L^{2}(I)\right)} \rightarrow 0 .
\end{aligned}
$$

By Proposition 2.1, there exists a $T_{*} \in(0,+\infty)$; therefore the initial boundary value problem

$$
\left\{\begin{array}{lr}
\rho_{t}+(\rho u)_{x}=0, & \\
(\rho u)_{t}+\left(\rho u^{2}\right)_{x}-\left(\left(u_{x}^{2}+\mu_{0}\right)^{\frac{p-2}{2}} u_{x}\right)_{x}+\pi_{x}=\rho f^{\delta} & (x, t) \in \Omega_{T}, \\
\left.(\rho, u)\right|_{t=0}=\left(\rho_{0}^{\delta}, u_{0}^{\delta}\right) & x \in[0,1], \\
\left.u\right|_{x=0}=\left.u\right|_{x=1}=0 & t \in[0, T], \\
\pi \equiv \pi(\rho)=A \rho^{\gamma}, \quad A>0, \quad \gamma>1, &
\end{array}\right.
$$

yields a unique solution $\left(\rho^{\delta}, u^{\delta}\right)$. Moreover, $\left(\rho^{\delta}, u^{\delta}\right)$ satisfies the following uniform estimate:

$$
\begin{aligned}
\operatorname{ess} \sup _{0 \leqslant t \leqslant T_{*}} & \left(\left|\rho^{\delta}(t)\right|_{H^{1}(I)}+\left|u^{\delta}(t)\right|_{W_{0}^{1, p}(I) \cap H^{2}(I)}+\left|\sqrt{\rho^{\delta}} u_{t}^{\delta}(t)\right|_{L^{2}(I)}+\left|\rho_{t}^{\delta}(t)\right|_{L^{2}(I)}\right) \\
& +\int_{0}^{T_{*}}\left|u_{x t}^{\delta}(s)\right|_{L^{2}(I)}^{2} \mathrm{~d} s \leqslant C,
\end{aligned}
$$

where $C$ is a positive constant, depending only on $M^{0}$. Then we obtain the following convergence:

$$
\begin{aligned}
& u^{\delta} \rightarrow u \quad \text { in } \quad L^{\infty}\left(0, T_{*} ; L^{2}(I)\right) \cap L^{2}\left(0, T_{*} ; H_{0}^{1}(I)\right), \\
& \rho^{\delta} \rightarrow \rho \quad \text { in } \quad L^{\infty}\left(0, T_{*} ; L^{2}(I)\right) .
\end{aligned}
$$

Hence, similar to the proof of Proposition 2.1. we may prove that $(\rho, u)$ is the solution of the original problem (1.1)-(1.2); moreover, $(\rho, u)$ satisfies the following uniform estimate:

$$
\begin{gathered}
\operatorname{ess} \sup _{0 \leqslant t \leqslant T_{*}}\left(|\rho(t)|_{H^{1}(I)}+|u(t)|_{W_{0}^{1, p}(I) \cap H^{2}(I)}+\left|\sqrt{\rho} u_{t}(t)\right|_{L^{2}(I)}+\left|\rho_{t}(t)\right|_{L^{2}(I)}\right) \\
+\int_{0}^{T_{*}}\left|u_{x t}(s)\right|_{L^{2}(I)}^{2} \mathrm{~d} s \leqslant C
\end{gathered}
$$

where $C$ is a positive constant, depending only on $M^{0}$.

The proof of continuity of the solution of (1.1)-(1.2) is similar to the proof of Proposition 2.1. Therefore, the proof of existence of the main Theorem 1.2 is finished. 
3.2. The proof of uniqueness. Assume that $(\rho, u),(\bar{\rho}, \bar{u})$ are both solutions of (1.1)(1.2); then

$$
\begin{aligned}
& \int_{0}^{1}[\rho(x, t) u(x, t)-\bar{\rho}(x, t) \bar{u}(x, t)] \phi(x, t) \mathrm{d} x-\int_{0}^{t} \int_{0}^{1}\left\{(\rho u-\bar{\rho} \bar{u}) \phi_{t}+\left(\rho u^{2}-\bar{\rho} \bar{u}^{2}\right) \phi_{x}\right. \\
& \left.-\left(\left(u_{x}^{2}+\mu_{0}\right)^{\frac{p-2}{2}} u_{x}-\left(\bar{u}_{x}^{2}+\mu_{0}\right)^{\frac{p-2}{2}} \bar{u}_{x}\right) \phi_{x}+(\pi-\bar{\pi}) \phi_{x}\right\}(x, s) \mathrm{d} x \mathrm{~d} s \\
= & \int_{0}^{t} \int_{0}^{1}(\rho-\bar{\rho}) f \phi(x, s) \mathrm{d} x \mathrm{~d} s .
\end{aligned}
$$

By definition (1.5), we have

$$
\begin{aligned}
0= & \int_{0}^{t} \int_{0}^{1}\left\{(u-\bar{u}) \bar{u}_{x}((\rho-\bar{\rho}) \bar{u}-(\rho u-\bar{\rho} \bar{u})+\rho(u-\bar{u}))\right\}(x, s) \mathrm{d} x \mathrm{~d} s \\
= & \int_{0}^{t} \int_{0}^{1}\left\{\bar{u}_{t}(\rho-\bar{\rho})(u-\bar{u})-(\rho-\bar{\rho}) \bar{u} \bar{u}_{x}(u-\bar{u})-\rho(u-\bar{u})^{2} \bar{u}_{x}\right. \\
& \left.+(\rho-\bar{\rho}) \bar{u}(u-\bar{u})_{t}+(\rho u-\bar{\rho} \bar{u}) \bar{u}(u-\bar{u})_{x}\right\}(x, s) \mathrm{d} x \mathrm{~d} s+\int_{0}^{1}(\rho-\bar{\rho}) \bar{u}(u-\bar{u})(x, t) \mathrm{d} x \\
= & \int_{0}^{t} \int_{0}^{1}\left\{(\rho-\bar{\rho})\left(\bar{u}_{t}-\bar{u} \bar{u}_{x}\right)(u-\bar{u})+\rho(u-\bar{u})^{2} \bar{u}_{x}+(\rho-\bar{\rho}) \bar{u}(u-\bar{u})_{t}\right. \\
& \left.+(\rho u-\bar{\rho} \bar{u}) \bar{u}(u-\bar{u})_{x}\right\}(x, s) \mathrm{d} x \mathrm{~d} s+\int_{0}^{1}(\rho-\bar{\rho}) \bar{u}(u-\bar{u})(x, t) \mathrm{d} x .
\end{aligned}
$$

Let

$$
\Psi(s)=\left(s^{2}+\mu_{0}\right)^{\frac{p-2}{2}} s .
$$

Then

$$
\int_{0}^{1}\left[\mathrm{E}_{p} u-\mathrm{E}_{p} \bar{u}\right](u-\bar{u})_{x} \mathrm{~d} x=\int_{0}^{1}\left[\int_{0}^{1} \Psi^{\prime}(\theta u+(1-\theta) \bar{u}) \mathrm{d} \theta\right]\left(u_{x}-\bar{u}_{x}\right)^{2} \mathrm{~d} x,
$$

and since

$$
\begin{aligned}
\Psi^{\prime}(s) & =\left[\left(s^{2}+\mu_{0}\right)^{\frac{p-2}{2}} s\right]^{\prime} \\
& =(p-2)\left(s^{2}+\mu_{0}\right)^{\frac{p-4}{2}} s^{2}+\left(s^{2}+\mu_{0}\right)^{\frac{p-2}{2}} \\
& =\left(s^{2}+\mu_{0}\right)^{\frac{p-4}{2}}\left((p-1) s^{2}+\mu_{0}\right) \\
& \geqslant\left(s^{2}+\mu_{0}\right)^{\frac{p-2}{2}} \\
& \geqslant \mu_{0}^{\frac{p-2}{2}}
\end{aligned}
$$


then (3.70) may be written as

$$
\int_{0}^{1}\left[\mathrm{E}_{p} u-\mathrm{E}_{p} \bar{u}\right](u-\bar{u})_{x} \mathrm{~d} x \geqslant C \int_{0}^{1}\left(u_{x}-\bar{u}_{x}\right)^{2} \mathrm{~d} x .
$$

In (3.68), we take $\phi=(u-\bar{u}) \in C\left([0, T] ; H_{0}^{1}(I)\right)$. Substituting (3.71) into (3.68), and using (3.69), we have

$$
\begin{aligned}
& \frac{1}{2} \int_{0}^{1} \rho(u-\bar{u})^{2}(x, t) \mathrm{d} x+\int_{0}^{t} \int_{0}^{1}\left(\left(u_{x}^{2}+\mu_{0}\right)^{\frac{p-2}{2}} u_{x}-\left(\bar{u}_{x}^{2}+\mu_{0}\right)^{\frac{p-2}{2}} \bar{u}_{x}\right)(u-\bar{u})_{x} \mathrm{~d} x \mathrm{~d} s \\
= & \int_{0}^{t} \int_{0}^{1}\left\{(\rho-\bar{\rho})\left(f+\bar{u}_{t}-\bar{u} \bar{u}_{x}\right)(u-\bar{u})+\rho(u-\bar{u})^{2} \bar{u}_{x}+(\pi-\bar{\pi})(u-\bar{u})_{x}\right\}(x, t) \mathrm{d} x \mathrm{~d} s \\
\leqslant & \int_{0}^{t} \int_{0}^{1}\left[|\rho-\bar{\rho}||h||u-\bar{u}|+\rho|u-\bar{u}|^{2}\left|\bar{u}_{x}\right|+|\pi-\bar{\pi}|\left|u_{x}-\bar{u}_{x}\right|\right] \mathrm{d} x \mathrm{~d} s \\
\leqslant & \int_{0}^{t}\left\{|\rho-\bar{\rho}|_{L^{2}(I)}|h|_{L^{2}(I)}|u-\bar{u}|_{L^{\infty}(I)}+|\sqrt{\rho}(u-\bar{u})|_{L^{2}(I)}^{2}\left|\bar{u}_{x}\right|_{L^{\infty}(I)}\right. \\
& \left.+|\pi-\bar{\pi}|_{L^{p}(I)}\left|u_{x}-\bar{u}_{x}\right|_{L^{2}(I)}\right\} \mathrm{d} s \\
\leqslant & C \int_{0}^{t}\left\{|(\rho-\bar{\rho})(s)|_{L^{2}(I)}^{2}|h(s)|_{L^{2}(I)}^{2}+\frac{1}{4}\left|\left(u_{x}-\bar{u}_{x}\right)(s)\right|_{L^{2}(I)}^{2}\right. \\
& \left.+|\sqrt{\rho}(u-\bar{u})(s)|_{L^{2}(I)}^{2}\left|\bar{u}_{x}(s)\right|_{L^{\infty}(I)}+C|(\pi-\bar{\pi})(s)|_{L^{2}(I)}^{2}+\frac{1}{4}\left|\left(u_{x}-\bar{u}_{x}\right)(s)\right|_{L^{2}(I)}^{2}\right\} \mathrm{d} s
\end{aligned}
$$

where $h=\left(f+\bar{u}_{t}-\bar{u} \cdot \bar{u}_{x}\right) \in L^{2}\left(0, T ; L^{2}(I)\right)$.

Then we obtain

$$
\begin{aligned}
& \quad|\sqrt{\rho}(u-\bar{u})(t)|_{L^{2}(I)}^{2}+C \int_{0}^{t}\left|\left(u_{x}-\bar{u}_{x}\right)(s)\right|_{L^{2}(I)}^{2} \mathrm{~d} s \\
& \leqslant \int_{0}^{t}\left[C|(\rho-\bar{\rho})(s)|_{L^{2}(I)}^{2}|h(s)|_{L^{2}(I)}^{2}+|\sqrt{\rho}(u-\bar{u})(s)|_{L^{2}(I)}^{2}\left|\bar{u}_{x}(s)\right|_{L^{\infty}(I)}\right. \\
& \left.\quad+C|(\pi-\bar{\pi})(s)|_{L^{2}(I)}^{2}\right] \mathrm{d} s \\
& \leqslant \int_{0}^{t} A(s)\left[|\sqrt{\rho}(u-\bar{u})(s)|_{L^{2}(I)}^{2}+|(\rho-\bar{\rho})(s)|_{L^{2}(I)}^{2}+|(\pi-\bar{\pi})(s)|_{L^{2}(I)}^{2}\right] \mathrm{d} s,
\end{aligned}
$$

where the nonnegative function $A(t)=C\left(1+|h(s)|_{L^{2}(I)}^{2}+\left|\bar{u}_{x}(s)\right|_{L^{\infty}(I)}^{2}\right) \in L^{1}\left(0, T_{*}\right)$, and $C$ is a positive constant, depending only on $M^{0}$.

In (1.5), we take $\varphi=(\rho-\bar{\rho}) \in C\left([0, T] ; H^{1}(I)\right)$; then

$$
\frac{1}{2} \int_{0}^{1}(\rho-\bar{\rho})^{2} \mathrm{~d} x=\int_{0}^{t} \int_{0}^{1}(\rho-\bar{\rho})(\rho-\bar{\rho})_{t} \mathrm{~d} x \mathrm{~d} s+\int_{0}^{t} \int_{0}^{1}(\rho u-\bar{\rho} \bar{u})(\rho-\bar{\rho})_{x} \mathrm{~d} x \mathrm{~d} s .
$$


So, we have

$$
\begin{aligned}
& \frac{1}{2} \int_{0}^{1}(\rho-\bar{\rho})^{2}(t) \mathrm{d} x \\
= & \int_{0}^{t} \int_{0}^{1}(\rho u-\bar{\rho} \bar{u})_{x}(\rho-\bar{\rho}) \mathrm{d} x \mathrm{~d} s \\
= & \int_{0}^{t} \int_{0}^{1}(\rho u-\rho \bar{u}+\rho \bar{u}-\bar{\rho} \bar{u})_{x}(\rho-\bar{\rho}) \mathrm{d} x \mathrm{~d} s \\
= & \int_{0}^{t} \int_{0}^{1}\left(\rho_{x}(u-\bar{u})(\rho-\bar{\rho})+\rho(u-\bar{u})_{x}(\rho-\bar{\rho})+\frac{1}{2} \bar{u}_{x}(\rho-\bar{\rho})^{2}\right) \mathrm{d} x \mathrm{~d} s \\
\leqslant & \int_{0}^{t}\left(\left|\rho_{x}\right|_{L^{2}(I)}|u-\bar{u}|_{L^{\infty}}|\rho-\bar{\rho}|_{L^{2}(I)}+|\rho|_{L^{\infty}(I)}\left|u_{x}-\bar{u}_{x}\right|_{L^{2}}|\rho-\bar{\rho}|_{L^{2}(I)}\right. \\
& \left.+\frac{1}{2}\left|\bar{u}_{x}\right|_{L^{\infty}(I)}|\rho-\bar{\rho}|_{L^{2}(I)}^{2}\right) \mathrm{d} s \\
\leqslant & \int_{0}^{t}\left(B(s)|(\rho-\bar{\rho})(s)|_{L^{2}(I)}^{2}+\frac{1}{8}\left|\left(u_{x}-\bar{u}_{x}\right)(s)\right|_{L^{2}(I)}^{2}\right) \mathrm{d} s,
\end{aligned}
$$

where the nonnegative function $B(t)=C\left(|\rho|_{H^{1}(I)}+\left|\bar{u}_{x}\right|_{L^{\infty}(I)}\right) \in L^{1}\left(0, T_{*}\right)$, and $C$ is a positive constant, depending only on $M^{0}$.

Similarly, we have

$$
\int_{0}^{t} \frac{1}{2} \frac{\mathrm{d}}{\mathrm{d} t} \int_{0}^{1}(\pi-\bar{\pi})^{2} \mathrm{~d} x \mathrm{~d} s \leqslant \int_{0}^{t}\left(D(s)|(\pi-\bar{\pi})(s)|_{L^{2}(I)}^{2}+\frac{1}{8}\left|\left(u_{x}-\bar{u}_{x}\right)(s)\right|_{L^{2}(I)}^{2}\right) \mathrm{d} s,
$$

where the nonnegative function $D(t)=C\left(|\pi|_{H^{1}(I)}+\left|\bar{u}_{x}\right|_{L^{\infty}(I)}\right) \in L^{1}\left(0, T_{*}\right)$, and $C$ is a positive constant, depending only on $M^{0}$.

Combining (3.72)-(3.74), we obtain

$$
\begin{aligned}
& \frac{1}{4} \int_{0}^{t}\left|\left(u_{x}-\bar{u}_{x}\right)(s)\right|_{L^{2}(I)}^{2} \mathrm{~d} s+\left[|\sqrt{\rho}(u-\bar{u})(t)|_{L^{2}(I)}^{2}+|(\rho-\bar{\rho})(t)|_{L^{2}(I)}^{2}+|(\pi-\bar{\pi})(t)|_{L^{2}(I)}^{2}\right] \\
& \leqslant \int_{0}^{T_{*}}(A(s)+B(s)+D(s))\left(|\sqrt{\rho}(u-\bar{u})(s)|_{L^{2}(I)}^{2}+|(\rho-\bar{\rho})(s)|_{L^{2}(I)}^{2}\right. \\
& \left.\quad+|(\pi-\bar{\pi})(s)|_{L^{2}(I)}^{2}\right) \mathrm{d} s .
\end{aligned}
$$

By Gronwall's inequality, we have

$$
\begin{aligned}
\frac{1}{4}\left|\left(u_{x}-\bar{u}_{x}\right)(t)\right|_{L^{2}(I)}^{2} & +\operatorname{ess} \sup _{0 \leqslant t \leqslant T_{*}}\left(|\sqrt{\rho}(u-\bar{u})(t)|_{L^{2}(I)}^{2}+|(\rho-\bar{\rho})(t)|_{L^{2}(I)}^{2}\right. \\
& \left.+|(\pi-\bar{\pi})(t)|_{L^{2}(I)}^{2}\right) \leqslant 0 .
\end{aligned}
$$

Then we get

$$
\rho=\bar{\rho}, \quad \sqrt{\rho}(u-\bar{u})=0, \quad u_{x}=\bar{u}_{x},
$$


and $u(x, t) \in C\left(\left[0, T_{*}\right] ; H_{0}^{1}(I)\right)$. So,

$$
\rho=\bar{\rho}, \quad u=\bar{u} .
$$

Hence, the proof of uniqueness of the main theorem 1.2 is finished.

Therefore, Theorem 1.2 is proved.

\section{REFERENCES}

[1] O. A. Ladyzhenskaya, New equations for the description of the viscous incompressible fluids and solvability in the large of the boundary value problems for them, in "Boundary Value Problems of Mathematical Physics V", Amer. Math. Soc., Providence, RI, 1970.

[2] S. Whitaker, "Introduction to fluid mechanics", Krieger, Melbourne, FL, 1986.

[3] J. Nečas, M. Šilhavý, Multipolar viscous fluids, Quarterly of Applied Mathematics, 1991, Vol.XLIX(2), 247-265. MR.1106391 (92d:76005)

[4] H. Bellout, F. Bloom, J. Nečas, Phenomenological behavior of multipolar viscous fluids, Quarterly of Applied Mathematics, 1992, Vol.L(3), 559-583. MR1178435 (93g:76006)

[5] J. Málek, J. Nečas, M. Rokyta, M. Rǔžička, Weak and Measure-valued Solutions to Evolutionary PDEs, Chapman and Hall, New York, 1996. MR:1409366 (97g:35002)

[6] Š. Nečasová, M. Lukáčová, Bipolar isothermal non-Newtonian compressible fluids, Journal of Mathematical Analysis and Applications, 1998, Vol.225, 168-192. MR1639232 (99f:76008)

[7] Š. Nečasová, M. Lukáčová, Bipolar barotropic non-Newtonian fluid, Comment. Math. Univ. Carolinae, 1994, Vol. 35(3), 467-483. MR1307274 (95m:76007)

[8] J. Nečas, A. Novotný, Some qualitative properties of the viscous compressible heat conductive multipolar fluid, Communication in Partial Differential Equations, 1991, Vol.16(2\&3), 197-220. MR:1104099 (92m:76016)

[9] J. Nečas, A. Novotný, M. Šilhavý, Global solution to the compressible isothermal multipolar fluid, Journal of Mathematical Analysis and Applications, 1991, Vol.162, 223-241. MR1135273 (93e:35089)

[10] Hyeong-Ohk Bae, Existence, regularity, and decay rate of solutions of non-Newtonian flow, Journal of Mathematical Analysis and Applications, 1999, Vol.231, 467-491. MR.1669171 (99m:35194)

[11] R. Salvi, I. Straskraba, Global existence for viscous compressible fluids and their behavior as $t \rightarrow \infty$, J. Fac. Sci. Univ. Tokyo Sect. IA, Math., 1993, Vol.40, 17-51. MR1217657 (94f:35112)

[12] Hi Jun Choe, Hyunseok Kim, Strong solutions of the Navier-Stokes equations for isentropic compressible fluids, Journal of Differential Equations, 2003, Vol.190, 504-523. MR1970039 (2004b:35258)

[13] Yonggeun Cho, Hi Jun Choe, Hyunseok Kim, Unique solvability of the initial boundary value problems for compressible viscous fluids, Journal Mathematics Pure and Applications, 2004, Vol.83, 243-275. MR2038120(2005a:76133)

[14] T.-P. Liu, T. Yang, Compressible Euler equations with vacuum, J. Differential Equations, 1997, Vol.140, 223-237. MR1483001 (99a:35202)

[15] T.-P. Liu, Z. Xin, T. Yang, Vacuum states of compressible flow, Discrete and Continuous Dyn. Systems 1998, vol.4, 1-32. MR 1485360 (98k:76116)

[16] T. Makino, S. Ukai, S. Kawashima, Sur la solution a support compact de l' equation d'Euler compressible, Japan, J. Appl. Math. 1986, 3, 249-257. MR899222 (88g:35159)

[17] O. A. Ladyzhenskaya, V. A. Solonnikov, N. N. Ural'ceva, Linear and quasi-linear equations of parabolic type, Amer. Math. Soc., Providence, RI, 1968.

[18] G. P. Galdi, An introduction to the mathematical theory of the Navier-Stokes equations, SpringerVerlag, New York, 1994.

[19] O. A. Ladyzhenskaya, Boundary Value Problems of Mathematical Physics, Springer-Verlag, New York, 1985. MR793735 (87f:35001)

[20] R. Temam, Navier-Stokes equations: Theory and numerical analysis, North-Holland, Amsterdam, 1977. MR0609732 (58:29439) 